\title{
Vildagliptin Attenuates Huntington's Disease through Activation of GLP-1 Receptor/PI3K/Akt/BDNF Pathway in 3-Nitropropionic Acid Rat Model
}

\author{
Noha H. Sayed ${ }^{1} \cdot$ Nevine Fathy $^{1}$ (D) $\cdot$ Mona A. Kortam ${ }^{1} \cdot$ Mostafa A. Rabie $^{2} \cdot$ Ahmed F. Mohamed $^{2} \cdot$ Ahmed S. Kamel $^{2}$
}

Published online: 14 November 2019

(C) The American Society for Experimental NeuroTherapeutics, Inc. 2019, corrected publication 2022

\begin{abstract}
Vildagliptin (Vilda), a dipeptidyl peptidase-4 (DPP-4) inhibitor, has been highlighted as a promising therapeutic agent for neurodegenerative diseases as Alzheimer's and Parkinson's diseases. Vilda's effect is mostly linked to PI3K/Akt signaling in CNS. Moreover, $\mathrm{PI} 3 \mathrm{~K} /$ Akt activation reportedly enhanced survival and dampened progression of Huntington's disease (HD). However, Vilda's role in $\mathrm{HD}$ is yet to be elucidated. Thus, the aim of the study is to uncover the potentiality of Vilda in HD and unfold its link with PI3K/Akt pathway in 3-nitropropionic acid (3NP) rat model. Rats were randomly assigned into 4 groups; group 1 received saline, whereas, groups 2, 3 and 4 received 3NP (10 mg/kg/day; i.p.) for 14 days, concomitantly with Vilda (5 mg/kg/day; p.o.) in groups 3 and 4, and wortmannin (WM), a PI3K inhibitor, $(15 \mu \mathrm{g} / \mathrm{kg} /$ day; i.v.) in group 4 . Vilda improved cognitive and motor perturbations induced by $3 \mathrm{NP}$, as confirmed by striatal histopathological specimens and immunohistochemical examination of GFAP. The molecular signaling of Vilda was estimated by elevation of GLP-1 level and protein expressions of survival proteins; p85/p55 (pY458/199)-PI3K, $p$ S473Akt. Together, it boosted striatal neurotrophic factors and receptor; $p$ S133-CREB, BDNF, $p$ Y515-TrKB, which subsequently maintained mitochondrial integrity, as indicated by enhancing both SDH and COX activities, and the redox modulators; Sirt1, Nrf2. Such neuroprotection restored imbalance of neurotransmitters through increasing GABA and suppressing glutamate as well PDE10A. These effects were reversed by WM pre-administration. In conclusion, Vilda purveyed significant anti-Huntington effect which may be mediated, at least in part, via activation of GLP-1/PI3K/Akt pathway in 3NP rat model.
\end{abstract}

Key Words 3-nitropropionic acid · Mitochondrial dysfunction · Glucagon like peptide-1 $\cdot \mathrm{PI}_{3} \mathrm{~K} / \mathrm{Akt}$ signaling $\cdot$ Vildagliptin

\section{Introduction}

Huntington's disease (HD) is a fatal neurodegenerative disease characterized by striatal degeneration leading to motor, psychiatric, and cognitive impairments for which there is no cure and whose progression cannot be slowed down or reversed $[1,2]$. The causes of neuronal degeneration are not completely understood, but oxidative stress, mitochondrial dysfunction and changes in energy metabolism are regarded as the main contributors to the pathophysiology of HD [2].

Nevine Fathy

nevine.abdallah@pharma.cu.edu.eg

1 Department of Biochemistry, Faculty of Pharmacy, Cairo University, Governorate, Giza, Egypt

2 Department of Pharmacology and Toxicology, Faculty of Pharmacy, Cairo University, Governorate, Giza, Egypt
One of the endogenous hormones that attracts the consideration in the recent research is glucagon-like peptide-1 (GLP1) that emerged as a guard against neurodegenerative diseases in different experimental models [3, 4]. However, one major imperfection of GLP-1 was the short half-life attributed to degradation by dipeptidyl peptidase-4 enzyme (DPP-4). This promoted the fructification of DPP-4 inhibitors (gliptins), a relatively new class of oral diabetes drugs that are dependent on the elevation of GLP-1 level. Accordingly, binding of GLP-1 to its receptor (GLP-1R) activates phosphoinositide 3-kinase (PI3K) with subsequent enhancement of CREB expression leading to cellular proliferation, mitochondrial biogenesis, inhibition of apoptotic and inflammatory processes that ultimately ends up with enhanced cell survival [5].

Various DPP-4 inhibitors showed a prominent effect in several pre-clinical and clinical trials for the treatment of neurodegenerative disorders [6-8]. One of the potent and selective DPP-4 inhibitors is Vildagliptin (Vilda) that is approved 
as an anti-hyperglycemic drug for the treatment of type- 2 diabetes [9]. Relatively, recent studies pointed to the neuroprotective effect of Vilda against Alzheimer's disease (AD) and Parkinson's disease (PD) which is ascribed to activation of diverse signaling pathways by GLP-1R after DPP-4 inhibition $[10,11]$. The GLP-1 and PI3K are demonstrated to be the keys behind the neuroprotective effect of Vilda in different disorders [5]. Together, Chong et al. (2012) showed the indispensable role of PI3K and Akt proteins as cellular components in hindering HD [12].

However, as of yet, no data exist on the potential impact of Vilda in treatment of HD and its respective intracellular signal transduction which presents as a promising avenue for therapeutic intervention in this striatal neurodegenerative disorder. Herein, the current study aimed to investigate the possible neuroprotective effect of Vilda against 3-nitropropionic acid (3NP)-induced HD in rats and to unveil some of the possible involved signals through using wortmannin (WM) as a direct inhibitor of the PI3K pathway.

\section{Material and Methods}

\section{Ethics Statement}

The study protocol complies with the Guide for the Care and Use of Laboratory Animals protocol (NIH publication No. 85-23, revised 2011) implemented by the Ethics Research Committee of Faculty of Pharmacy, Cairo University (Cairo, Egypt; PT BC2403). All efforts were done to reduce animal suffering during the experimental period.

\section{Animals}

Adult male Wistar albino rats weighing $(180 \pm 20 \mathrm{~g})$ were obtained from the animal facility of Faculty of Pharmacy, Cairo University (Cairo, Egypt). Animals were allowed to acclimatize to laboratory conditions for 2 weeks before the experiment. Rats were kept under controlled environmental condition of constant temperature $\left(23 \pm 2{ }^{\circ} \mathrm{C}\right)$, humidity (60 $\pm 10 \%)$, and light/dark (12/12 h) cycle (lights on at 6:00 am). Rats were allowed free access to standard chow pellet and water ad libitum and all behavioral tests were carried out in a sound isolated laboratory.

\section{Experimental Design}

Rats were randomly allocated into four groups; Group I received normal saline and served as control group. Group II received 3NP (10 mg/kg/day; i.p; Sigma-Aldrich, MO, USA) [13]. Group III treated with Vilda $(5 \mathrm{mg} / \mathrm{kg}$ /day; p.o.; Novartis International AG, Switzerland) $1 \mathrm{~h}$ after 3NP injection [14]. Group IV treated with WM $(15 \mu \mathrm{g} / \mathrm{kg} /$ day; i.v.; Sigma-Aldrich, MO, USA) 15 min prior Vilda administration [15]. All treatments were conducted for 14 days; where, 3NP, as well as, Vilda was dissolved in saline, and WM was dissolved in dimethyl sulfoxide (Merck, Germany) and freshly diluted with saline. Each group consisted of 18 rats that were subjected to behavioral analysis as shown in Scheme 1. Blood samples were collected from retroorbital sinus of all rats for evaluation of serum glucose and GLP1 levels. Animals were further classified into 4 subsets; first subset $(n=5)$ was used to assess parameters by Western blot technique, second subset $(n=5)$ was used to measure parameters by ELISA technique, third subset $(n=5)$ was used for mitochondrial isolation and succinate dehydrogenase (SDH) and cytochrome c oxidase (COX) activity assay,whereas, the last subset $(n=3)$ was used for striatal histopathological examination and immunohistochemical assessment of glial fibrillary acidic protein (GFAP), keeping into consideration blind unbiased slide reading. During the analysis of these measurements, the investigators were blinded to sample identity, and sample coding and decoding were performed by an independent experimenter.

\section{Behavioral Tests}

Twenty four hours after the last injection of 3NP, Vilda, and/or $\mathrm{WM}$, rats were screened for motor performance using the open field; rotarod, and grip strength meter analysis.

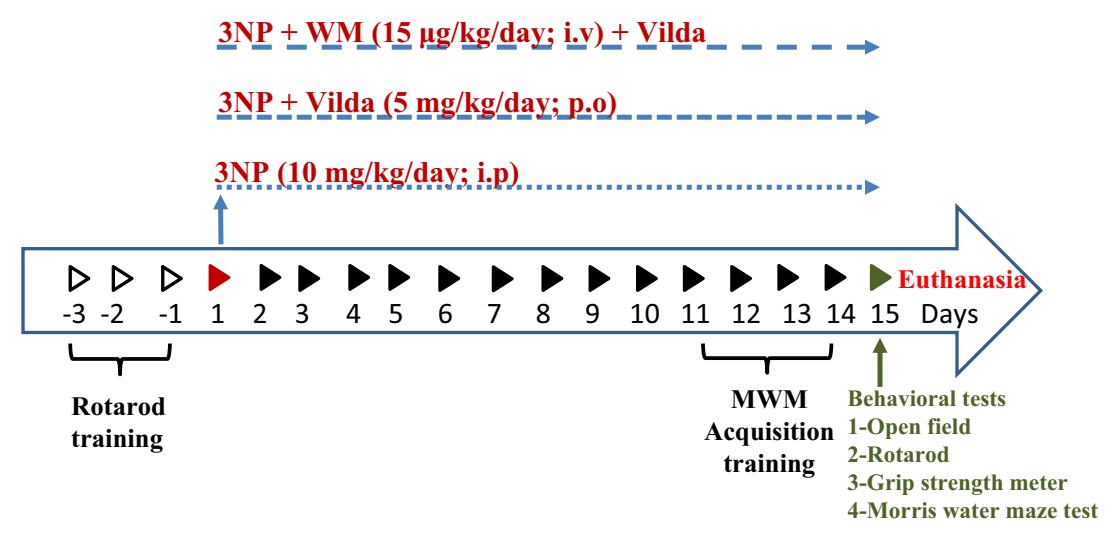

Scheme 1 Time schedule for vildagliptin and wortmannin and behavioral assessment in 3-NP rat model 
Additionally, spatial learning and memory were assessed using Morris water maze test. Tests were carried out in the same sequence with $2 \mathrm{~h}$ resting period between the tests [16]. Monitoring of animals' movement in the open field and Morris water maze tests was performed using ANY-Maze video tracking software (Stoelting Co, USA).

\section{Open Field Test (OFT)}

Open field test was performed to evaluate the animal's spontaneous locomotor activity. The test was carried out using a square wooden box $(80 \times 80 \times 40 \mathrm{~cm})$ with red walls and white polished floor divided by black lines into 16 equal squares. The test was performed under dim white light in a sound isolated room and an overhead camera was used to monitor the animals. Briefly, each rat was gently placed in the center of the open field and was allowed to freely explore the area for $180 \mathrm{~s}$, then the floor was cleaned after each tested animal. Rearing frequency, total distance traveled, mean speed, time immobile, as well as, distance traveled and time spent in the 4 central squares and peripheral 12 squares were recorded [17].

\section{Rotarod Test}

Rats were screened for motor coordination and balance using rotarod apparatus $(120 \mathrm{~cm}$ long and $3 \mathrm{~cm}$ in diameter and rotating at a constant speed of $20 \mathrm{rpm}$ ). Before experimental procedures, five trials were given to each rat; animals that continued on the rod for $5 \mathrm{~min}$ were selected to carry out the experiment. After completing OFT, the test was performed again where the fall off latency was recorded [18].

\section{Grip Strength Test}

The rat's forelimbs muscle strength was automatically measured using grip strength meter (Ugo Basile, Italy). Each rat was allowed to grasp a trapeze-shaped steel bar (stretched horizontally at a suitable height over a cushion support) mounted on a force gauge with its forepaws. The gauge was reset to zero after stabilization and the rat was gently pulled by the tail till the grasp was released. The peak force in gf was recorded, and the average of three consecutive measurements was calculated for each rat [19].

\section{Morris Water Maze}

Spatial learning and memory retention were examined using the Morris water maze. The maze consisted of a circular pool $(60 \mathrm{~cm}$ in height and $150 \mathrm{~cm}$ in diameter) filled with water up to $35 \mathrm{~cm}$ level and kept at a temperature of $25 \pm$ $2{ }^{\circ} \mathrm{C}$. The water was made opaque using a non-toxic watersoluble black paint. The pool was divided into 4 quadrants and a movable escape platform $(9 \mathrm{~cm}$ diameter) placed in the center of a specific quadrant. The pool was placed in a dimly lit room with fixed distal visual clues that served as navigational reference key for locating the target. In the acquisition phase, rats were subjected to 3 training sessions daily, each $120 \mathrm{~s}$, for 4 consecutive days. During each acquisition trial, animals were left free to find the platform in the target quadrant. Once the rat located the platform, it was permitted to remain on it for $10 \mathrm{~s}$, while if an animal failed to reach the platform within $120 \mathrm{~s}$, it was gently placed on the platform for $30 \mathrm{~s}$. The escape latency was calculated as the average of total time taken in all trials of each day of the acquisition phase to locate the platform and was used as a measure for spatial learning. On the fifth day, a probe test was performed where the platform was removed and each rat was placed in the water facing the pool wall starting from the quadrant opposite to the platform quadrant and was allowed to explore the pool for $60 \mathrm{~s}$. The time spent swimming in the target as well as opposite quadrants was recorded using an overhead camera [13].

\section{Striatal Processing}

At the end of behavioral tests, rats were euthanized and the brains were immediately dissected, striata from each brain were immediately isolated and flash frozen in liquid nitrogen, then stored at $-80^{\circ} \mathrm{C}$.

\section{Assessment of p85/p55 (pY458/199)-PI3K, pS473-Akt, pS133-CREB, BDNF, pY515-TrkB, Sirt1 and Nrf2}

In the first subset, tissues were washed in PBS and lysed by the Ready Prep ${ }^{\mathrm{TM}}$ protein extraction kit (Bio-Rad Inc., CA, USA). Protein levels were measured using Bradford protein assay kit (Thermo Fisher Scientific Inc., USA) [20] . Then, lysates were boiled for $5 \mathrm{~min}$ with Laemmli buffer, and proteins were separated by SDS-PAGE and transferred to an Immobilon membrane (Millipore). Protein expression was evaluated using primary antibodies (ThermoFisher Scientific, MA, USA) against p85/p55 ( $p$ Y458/199)-PI3K (1:1000; cat\#: PA5-17387), $p$ S473-Akt (1:250; cat\#: 700392), $p$ S133-CREB (1:250; cat\#: PA1-851B), BDNF (1:1000; cat\#: OSB00017W), $p$ Y515-TrkB (1:2500; cat\#: PA5-38076), Sirt1 (1:1000; cat\#: PA5-20964) and Nrf2 (1:1000; cat\#: PA5-67520). Antibodies were diluted in 5\% skimmed milk, Tris-HCl, $0.1 \%$ Tween 20, added to PVDF membranes containing specimen samples and incubated at $4 \mathrm{C}$ overnight. The membranes were then incubated with the appropriate secondary antibodies conjugated to horse-radish peroxidase for $2 \mathrm{~h}$ at room temperature. Densitometric analysis of the immunoblots was performed to quantify the amount of target proteins by Image analysis software on the ChemiDoc MP imaging system (version 3) produced by Bio-Rad (Hercules, CA). The same membranes were reprobed several times. Results are expressed as arbitrary units after normalization with $\beta$-actin protein expression. 
Quantification of Serum and Striatal GLP-1, As Well As, Striatal CAMP, PKA, GABA, Glutamate and PDE 10A

In the second subset, striata were homogenized in PBS (pH 7.4) and RayBio ELISA kit (GA, USA) was used for the determination of serum and striatal GLP-1 (cat\#: EIAGLP1, EIAM-GLP1, EIAR-GLP1), as well as, creative diagnostic ELISA kit (Shirley, NY, USA) was used for quantification of cAMP (Cat\#: DEIA2964). In addition, PKA activity (Cat\#: ab139435) was estimated using specific kit (Abcam, Cambridge, MA, USA). Also, a kit purchased from Elabscience (Wuhan, PRC) was used for quantification of GABA (cat\#: E0900r), whereas Abnova ELISA kit (Taipei, Taiwan) was used for assessment of glutamate (cat\#: KA1909). Likewise, PDE 10A (cat\#: MBS082585) was quantified with specific ELISA kit (MyBioSource Inc., San Diego, CA, USA). The procedures were performed according to the manufacturer's instructions. The results were presented as $\mathrm{pg} /$ $\mathrm{ml}$ for serum GLP-1, pg/mg protein for striatal GLP-1, $\mathrm{pmol} / \mathrm{mg}$ protein for cAMP, ng/min/mg protein for PKA, ng/ $\mathrm{mg}$ protein for PDE $10 \mathrm{~A}$ and $\mu \mathrm{g} / \mathrm{mg}$ protein for GABA and glutamate. The protein content of tissue homogenate was determined using the method of Lowry et al. [21]. Aliquots of the homogenate were used to assess oxidative stress biomarkers.

\section{Determination of Oxidative Stress Biomarkers}

Oxidative stress status was evaluated in striatal homogenate by measuring malondialdehyde (MDA), an end product of lipid peroxidation, colorimetrically using specific kit (Eagle Biosciences Inc., Boston, USA). In addition, reduced glutathione (GSH) quantification was performed according to the method of Ellman [22], whereas kit purchased from BioVision (CA, USA) was used to estimate superoxide dismutase (SOD) activity.

\section{Mitochondrial Isolation for SDH and COX Activity Assay}

Rat brain mitochondria were isolated using mitochondria isolation kit (Sigma-Aldrich Co., USA). The brain striatum was homogenized in isolation buffer containing $2 \mathrm{mg} / \mathrm{ml}$ albumin, $1 \mathrm{M}$ mannitol, $350 \mathrm{mM}$ sucrose, and $5 \mathrm{mM}$ Ethylene glycol tetraacetic acid (EGTA), $50 \mathrm{mM}$ 4-(2-hydroxyethyl)-1piperazineethanesulfonic acid (HEPES), $\mathrm{pH}$ 7.5. The homogenate was centrifuged at $600 \mathrm{x}$ g for $5 \mathrm{~min}$. The supernatant was carefully transferred to new tubes and centrifuged at $11,000 \mathrm{x} \mathrm{g}$ for $10 \mathrm{~min}$. The pellets were re-suspended in extraction buffer and spun again at $600 \mathrm{x} g$ for $5 \mathrm{~min}$. The resulting supernatant was transferred to new tubes and centrifuged at $11,000 \mathrm{x}$ g for $10 \mathrm{~min}$. The pellets containing pure mitochondria were resuspended in isolation buffer without
EGTA and used to assess SDH activity using the method described by Sharman and Bondy [23].

Briefly, assay mixture consist of potassium phosphate buffer, $100 \mathrm{mM}, \mathrm{pH} 7.5$, sodium succinate, $0.125 \mathrm{M}$, antimycin, $0.1 \mathrm{mg} / \mathrm{ml}, \mathrm{KCN}, 100 \mathrm{mM}$, ferricytochrome c, $350 \mathrm{mM}$, phenazine methosulfate, $3.3 \mathrm{mg} / \mathrm{ml}$. Succinate was oxidized by an artificial electron acceptor, phenazine methosulfate, reoxidation of the reduced phenazine methosulfate is accomplished by ferricytochrome-c, whose reduction is monitored spectrophotometrically. The reaction was started by the addition of the striatum mitochondrial sample, and the increase in absorbance of ferrocytochrome $\mathrm{c}$ at $551 \mathrm{~nm}$ minus the absorbance at $520 \mathrm{~nm}$ was measured during the first minute using Jenway 6405 spectrophotometer. Enzyme activity in nanomoles of succinate per minute per $\mathrm{mg}$ of protein was quantified from these absorbance data, using the extinction co-efficient $\left(9.0 \mathrm{mM}^{-1} \mathrm{~cm}^{-1}\right)$ of cytochrome c.

Activity of COX; Complex IV, was measured by oxidation of reduced cytochrome $\mathrm{c}$ in isolated mitochondria using cytochrome oxidase activity colorimetric assay kit (BioVision Inc., CA, USA). Measurements were conducted by strictly adhering to the manufacturers' instructions. Specifically, $10 \mu \mathrm{l}$ of the striatum mitochondrial sample was added to a designated sample well. The reaction was initiated by adding $120 \mu \mathrm{ldi}-$ luted cytochrome $\mathrm{c}$ to all wells. The absorbance was measured at $550 \mathrm{~nm}$ on the kinetic program over a period of $45 \mathrm{~min}$ at 5min interval. The rate of reaction was calculated by measuring the changes in $\mathrm{OD} / \mathrm{min}$ using the maximum linear rate. Results were expressed as $\mu \mathrm{mol}$ cytochrome c oxidized $/ \mathrm{min} /$ $\mathrm{mg}$ protein ( $\mathrm{U} / \mathrm{mg}$ protein), using molar extinction co-efficient of cytochrome c $\left(7.04 \mathrm{mM}^{-1} \mathrm{~cm}^{-1}\right)$.

\section{Serum Glucose Level}

Serum glucose level was quantified colorimetrically using specific glucose kit supplied by Spinreact (Girona, Spain) according to manufacturers' protocol.

Histopathological examination.

Striatal samples were fixed in $10 \%$ buffered formol saline for $24 \mathrm{~h}$. Samples were washed, dehydrated by serial dilutions of alcohol, cleared in xylene, and embedded in paraffin at $56{ }^{\circ} \mathrm{C}$ in hot air oven for $24 \mathrm{~h}$. Sections of $4 \mu \mathrm{m}$ were prepared and stained with hematoxylin and eosin (H\&E) and examined under light microscope [24]. For Nissl staining analysis, 4- $\mu \mathrm{m}$-thick paraffin-embedded sections were incubated with $1 \%$ cresyl violet at $50{ }^{\circ} \mathrm{C}$ for $20 \mathrm{~min}$. After rinsing with double distilled water, sections were dehydrated and mounted with Permount. Four random non-overlapping fields per tissue section were analyzed for quantification of intact cell number in striatum [25]. All micrographs were obtained using full HD microscope camera operated by Leica application module for tissue sections analysis (Leica Microsystems GmBH, Wetzlar, Germany). 
Immunohistochemistry and Optical Density of GFAP

Assessment of astroglial alteration was carried out using paraffin-embedded striatal sections. Samples were incubated with a rabbit polyclonal glial fibrillary acidic protein (GFAP) antibody (Genetex, CA, USA) for $60 \mathrm{~min}$ at $37^{\circ} \mathrm{C}$. Sections were washed with PBS and incubated for $60 \mathrm{~min}$ at $37^{\circ} \mathrm{C}$ with biotinylated secondary antibody (Dako, Carpenteria, CA, USA), and then with Avidin DH and biotinylated horseradish peroxidase $\mathrm{H}$ complex according to Vectastain $\mathrm{ABC}$ peroxidase kit instructions (Vector Laboratories Inc., Burlingame, CA, USA). Following another wash with PBS, the reaction was visualized with $3,3^{\prime}$-diaminobenzidine tetrahydrochloride (DAB Substrate Kit, Vector Laboratories Inc., Burlingame, CA, USA). Sections were counterstained with hematoxylin, dehydrated, and cleared in xylene then cover slipped for light microscopic examination. Quantification of GFAP was carried out by measuring the optical density from 4 randomly chosen fields in each section and averaged using image analysis software (Image J, version 1.46a, NIH, Bethesda, MD, USA) [24]. An experienced investigator, blinded to sample identity, has performed all histopathological assessments to avoid any bias.

\section{Statistical Analysis}

All data obtained were presented as mean \pm S.D. Results were analyzed using one-way analysis of variance test (one-way ANOVA) followed by Tukey's multiple comparison test for all parameters, except rearing frequency which was analyzed using Kruskal-Wallis test followed by Dunn's multiple comparison test. Statistical analysis was performed using GraphPad Prism software (version 6). For all statistical tests, the level of significance was fixed at $p<0.05$.

\section{Results}

\section{Effect of Vilda on Behavioral and Motor Alterations, As Well As, Body Weight in 3NP Rats}

Locomotor and behavioral deficits are main features of HD models, which indicate striatal degeneration and motor impairment. Herein, 3NP-intoxicated rats showed marked locomotor, motor coordination, muscle strength, as well as, cognitive deficits (Figs. 1 and 2). In the open field test, a significant reduction of the total distance traveled, mean speed and rearing frequency $(19.77 \%, 29.61 \%$ and $4.84 \%$, respectively). 3NP markedly suppressed both central and peripheral locomotor activity ( $13.87 \%$ and $20.83 \%$, respectively). This was coupled with a significant reduction in time spent in center $(31.84 \%)$ along with a marked increase in immobility time and time in periphery ( 3.08 folds and 1.11 folds, respectively) in the $3 \mathrm{NP}$ group. This was accompanied by a decline in fall off latency $(15.02 \%)$, peak force $(40.66 \%)$ in the rotarod and grip strength meter tests, respectively. In the Morris water maze test, spatial learning progression, indicated by escape latency in the acquisition phase, was increased in $3 \mathrm{NP}$ rats. In the probe trial, $3 \mathrm{NP}$ rats spent less time spent in the target quadrant $(56.9 \%)$ and more time in the opposite quadrant (2.19 folds). Post-treatment with Vilda effectively counteracted these effects, as manifested by the increase in total distance traveled ( 3.15 folds), mean speed ( 2.14 folds), rearing frequency (13.77 folds), distance in center (3.23 folds), distance in periphery ( 3.15 folds) and time in center ( 2.16 folds) together with the decline in immobility time and time in periphery ( $55.4 \%$ and $94.7 \%$, respectively) in the open field test. A concomitant increase in fall off latency (3.41 folds) and peak force (2.38 folds) was observed in Vildatreated rats. Vilda group also exhibited marked improvement in finding the hidden platform during the Morris water maze acquisition together with more time spent in target quadrant (1.46 folds) and less time in the opposite quadrant (70.93\%), compared to $3 \mathrm{NP}$ rats, in the probe test. Nevertheless, preadministration of WM largely abated Vilda's amendment of behavioral anomalies in open field, rotarod, grip strength meter, as well as, Morris water maze tests.

Regarding body weight, 3NP caused a significant weight loss of about $15 \%$ of normal values. Surprisingly, Vilda did not contribute to any further change in body weight, neither did WM pretreatment, relative to 3NP group (Table 1).

\section{Effect of Vilda on Striatal SDH and COX Activities, Sirt1 and Nrf2 Protein Expressions in 3NP Rats}

As well known in literature about the primary mechanism of toxicity of 3NP, 3NP showed significant dysfunction of the respiratory chain in mitochondria through reducing striatal SDH, COX activities, Sirt1 and Nrf2 to 26, 52, 54 and 55\% of control rats, respectively (Fig. 3). The respiratory function of mitochondria was significantly recovered in Vilda-treated group to increase by $1.86,1.88,1.38$ and 1.35 -folds, compared to 3NP treated group in respect to SDH, COX, Sirt1 and Nrf2. Meanwhile, this recovery was dampened in the WM pretreated group by $71,33,43$ and $62 \%$ reduction.

Effect of Vilda on GLP-1 and survival downstream protein alterations in $3 \mathrm{NP}$ rats.

To assess the possible Vilda-induced neuroprotective effect on striatal GLP-1 signaling, it was necessary to evaluate serum and striatal protein levels of GLP-1, as well as, striatal p-PI3K and p-Akt, cAMP and PKA kinase activity. In fig. 4, 3NP notably lowered both circulating and striatal levels of GLP-1 to $36 \%$ and $49 \%$ of control rats, concomitant with a reduction in protein expression of phosphorylated forms of PI3K and Akt by 55 and 50\%, as well as a decline in cAMP protein expression and PKA activity by $66 \%$ and $79 \%$, as compared to normal values. After Vilda 
(a)

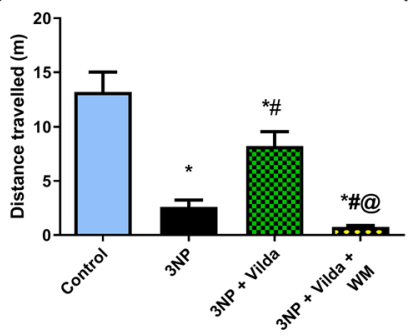

(b)

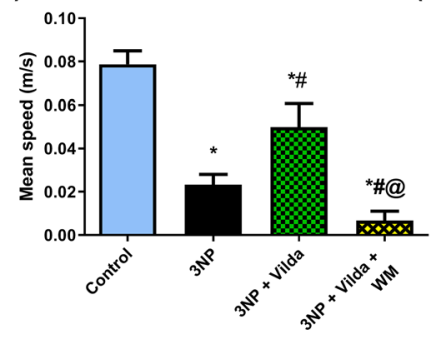

(f)

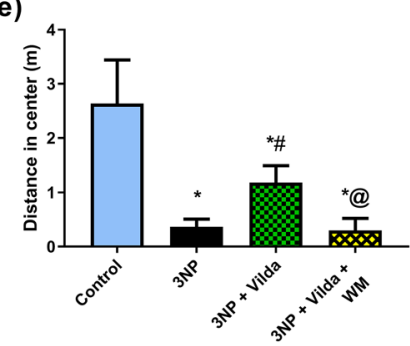

(i)
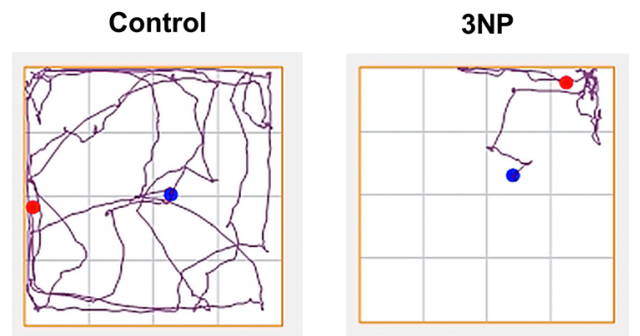

Fig. 1 Effect of Vilda on locomotor activity of 3NP rats in open field task. Panels represent total distance traveled (a), average speed (b), immobility time (c), rearing frequency (d), distance (e) and time (f) in center, distance $(\mathrm{g})$ and time $(\mathrm{h})$ in periphery as well as representative

treatment, the upstroke in circulating and striatal GLP-1 levels was significantly noted to reach 4.18 and 3 -fold of the model, and 1.49 and 1.5 -fold of normal rats, respectively. Subsequently, the protein expressions of p-PI3K and pAkt were elevated by 73 and $44 \%$ of $3 \mathrm{NP}$ treated group, in addition to 2.5 and 3.8-fold rise in cAMP protein expression and PKA activity. On the contrary, all these Vilda inducedperturbations were repealed with pre-administration of WM by 63 and $80 \%$ relative to serum and striatal GLP-1, and by $60,47,52$ and $71 \%$ relative to p-PI3K, p-Akt, cAMP and PKA, respectively. These results elaborated that Vilda exerted its neuroprotective action, in part, through the GLP-1 receptor/PI3K/Akt pathway.

\section{Effect of Vilda on PDE10A Level, in Addition to, Striatal Neuroplastic and Neurotrophic Factors Alterations in 3NP Rats}

PDE10A is a crucial regulator of cAMP signaling and, consequently, activation of the transcription factor CREB, and its (c)

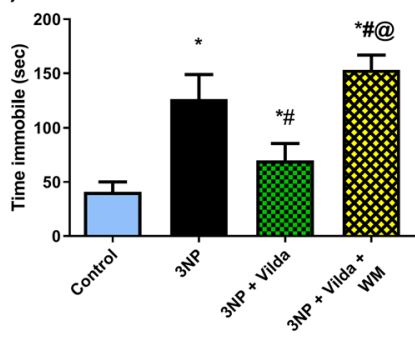

(d)

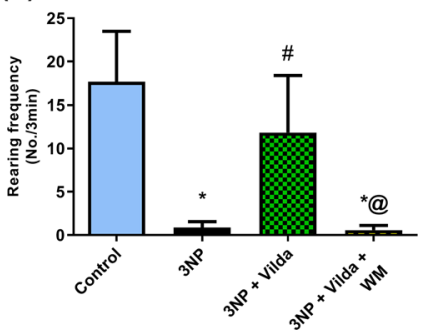

(g)

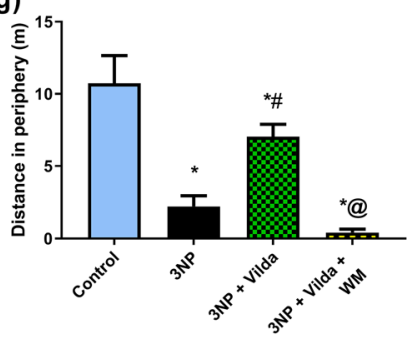

(h)

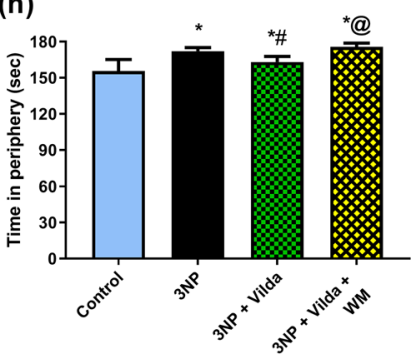

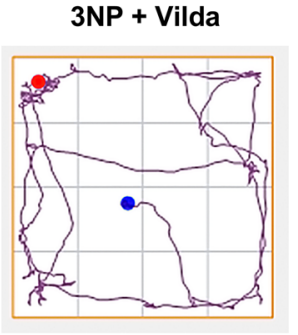

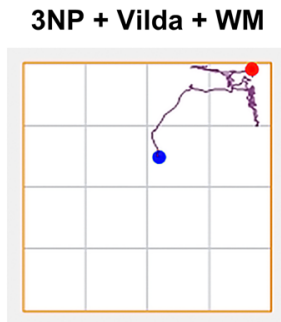

track plots (i) of rats during the test. Each bar with vertical line represents mean \pm S.D. of 18 rats per group. * vs control, \# vs 3NP, @ vs Vilda using one-way ANOVA followed by Tukey's post hoc test; $p<0.05$. 3NP; $3-$ nitropropionic acid, Vilda; vildagliptin, WM; wortmannin

downstream BDNF, a principal neurotrophic factor in striatum. Impairment of these signaling cascades is assumed to contribute to the pathology of HD. Thus, it was of interest to investigate the effects of Vilda on PDE10A inhibition, CREB phosphorylation (pCREB), BDNF and its receptor, tyrosine kinase $\mathrm{B}$ (TrkB) in the present HD model (Fig. 5). 3NP injection increased PDE10A level by 19-fold, while mitigated the protein expressions of $\mathrm{p}-\mathrm{CREB}, \mathrm{BDNF}$ and TrkB to reach 55, 52 and $54 \%$, in respect to $3 \mathrm{NP}$ free rats, respectively. Interestingly, Vilda abolished such elevation of PDE10A by $69 \%$ and amended the alterations of neuroplastic, neurotrophic proteins and TrkB receptor by 31,38 and $44 \%$ of Huntington's representative group, respectively. These findings with Vilda, however, were reverted in WM-pretreated rats and decreased to reach 52,59 and 43\%, regarding p-CREB, BDNF and TrkB receptor, respectively. Besides, it impeded the inhibitory effect of Vilda on PDE10A and increased its level 2.2-fold to return to the same level as the $3 \mathrm{NP}$ treatedgroup. Thus, PDE10 might be a good therapeutic target for Vilda to ameliorate HD-specific deficits. 


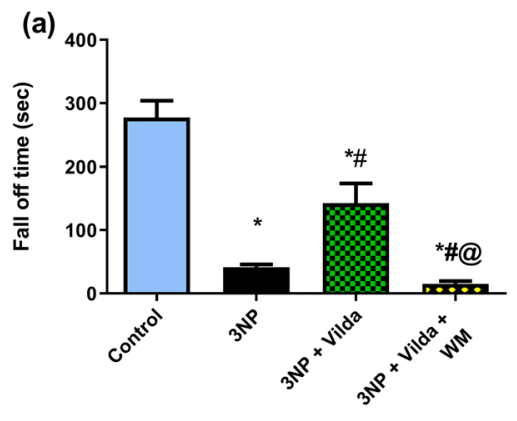

(c)

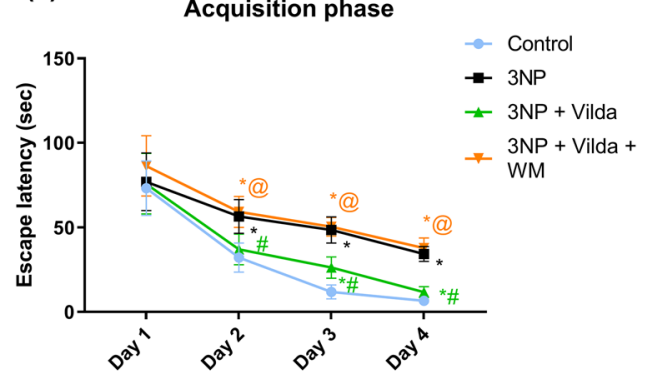

(e)
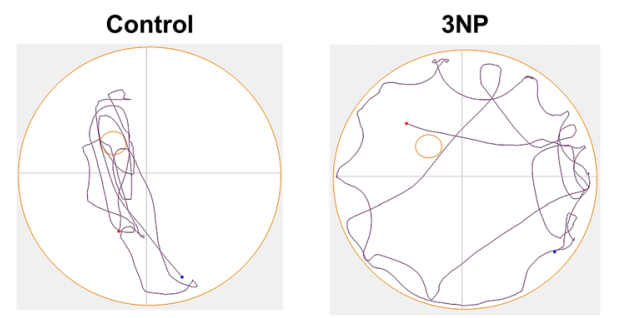

Fig. 2 Effect of Vilda on motor coordination, muscle strength, as well as cognitive deficits of $3 \mathrm{NP}$ rats in rotarod, grip strength meter and Morris water maze, respectively. Panels represent fall off latency (a) in rotarod, maximum peak force (b) in grip strength meter test, escape latency during acquisition (c), time spent in target/opposite quadrant (d)

\section{Effect of Vilda on GABA and Glutamate Levels in 3NP Rats}

Many of HD symptoms are associated with abnormal neurotransmission due to imbalance among inhibitory and excitatory neurotransmitters like GABA and glutamate. $3 \mathrm{NP}$ disturbed the balance between GABA and glutamate levels in striatum (Fig. 6). This is noted by the significant lowering of GABA and upleveling of glutamate by 74 and $48 \%$ in $3 \mathrm{NP}$ treated group, respectively. On the other hand, Vilda attenuated this blemish and significantly restored the levels of GABA and glutamate to reach 1.86 fold and $44 \%$ of Huntington's untreated group. However, WM-treated group blocked the Vilda-induced increase of GABA by $81 \%$ and decrease of glutamate by 1.7 -fold.

\section{Effect of Vilda on Oxidative Stress in 3NP Rats}

Interruption of the respiratory chain imparted by $3 \mathrm{NP}$, leads to increased production of ROS due to incomplete electron

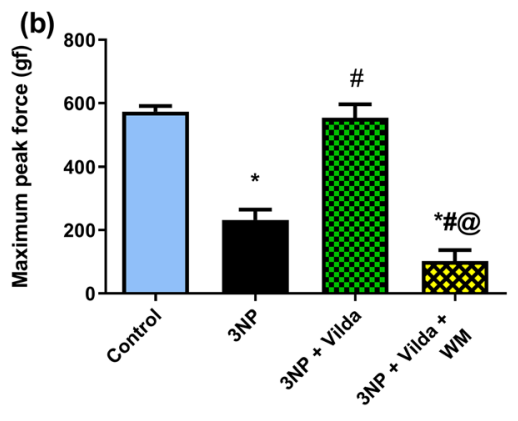

(d)

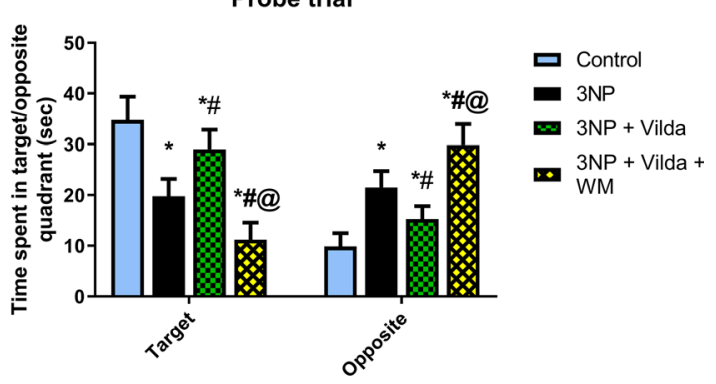

$3 \mathrm{NP}+$ Vilda

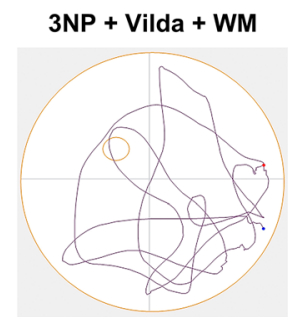

as well as representative swim paths (e) of rats during probe trial of Morris water maze test. Each bar with vertical line represents mean \pm S.D. of 18 rats per group. * vs control, \# vs 3NP, @ vs Vilda using one-way ANOVA followed by Tukey's post hoc test; $\mathrm{p}<0.05$. 3NP; 3-nitropropionic acid, Vilda; vildagliptin, WM; wortmannin

transfer, on one hand, and decreased antioxidant defense due to mitochondrial dysfunction, on the other hand. As shown in fig. 7, 3NP-induced oxidative damage is noted by the significant increase in the level of the lipid peroxidation product, MDA, by 12 -fold together with the significant decrease in the level of antioxidants, reduced GSH (72\%) and SOD activity

Table 1 Effect of Vilda on 3NP-induced alterations of body weight and serum glucose in rats

\begin{tabular}{llc}
\hline Group & Final body weight $(\mathrm{g})$ & Serum glucose $(\mathbf{m g} / \mathbf{d l})$ \\
\hline Control & $207.9 \pm 13.4$ & $98.4 \pm 10.9$ \\
3NP & $177.1 \pm 8.9^{*}$ & $159.6 \pm 13.4^{*}$ \\
3NP + Vilda & $175.6 \pm 7.6^{*}$ & $94.5 \pm 10.8^{\#}$ \\
3NP + Vilda + WM & $173.6 \pm 6.8^{*}$ & $132.5 \pm 8.1^{* \#}$ \\
\hline
\end{tabular}

Data are presented as means \pm S.D. of 18 rats per group. * vs control, \# vs 3NP, @ vs Vilda using one-way ANOVA followed by Tukey's post hoc test; $p<0.05$. 3NP; 3-nitropropionic acid, Vilda; vildagliptin, WM; wortmannin 
(57\%), in striatum as compared to control values. Vilda successfully alleviated this glitch and restored the levels of MDA, reduced GSH, and SOD activity to reach $22 \%, 287 \%$, and $255 \%$ of $3 \mathrm{NP}$ rats. WM pretreatment largely obliterated Vilda-induced modifications and caused a 2.74-fold rise in MDA level along with a reduction of $41 \%$ and $44 \%$ in reduced GSH level and SOD activity.

\section{Effect of Vilda on Blood Glucose in 3NP Rats}

Glucose metabolism is known to be altered in HD. To evaluate the effect of Vilda on glucose homeostasis in 3NP-induced HD model, serum glucose level was measured (Table 1). $3 \mathrm{NP}$ rats exhibited increased serum glucose levels that reached 1.6-fold of control values. Vilda treatment effectively normalized glucose levels, whereas pretreatment with WM hampered the normoglycemic effect of Vilda and increased serum glucose level by $40 \%$.

\section{Effect of Vilda on Striatal Histopathological Alterations in 3NP Rats}

HD is a neurodegenerative disorder which is characterized by degeneration of striatum; therefore, the protection of striatal neurons is a main goal of the protective agents. To validate the neuroprotection imparted by Vilda, histopathologic examination of hematoxylin-eosin, as well as, counting the number of intact neurons in striatum using cresyl violet was performed (Figs. 8 and 9). Control samples showed normal morphological features of striatum with apparent intact well-organized neurons showing vesicular nuclei and prominent nucleoli, as well as, normal neuropil with few glial cells infiltrations. Contrariwise, photomicrographs from 3NP rats demonstrated central core lesion with marked neuronal loss and many degenerated neurons with pyknotic nuclei, evidenced by a $53 \%$ decrease in viable neurons, accompanied by vacuolation and edema of neuropil with much microglial cell infiltration. Vilda-treated rats, however, showed (a)

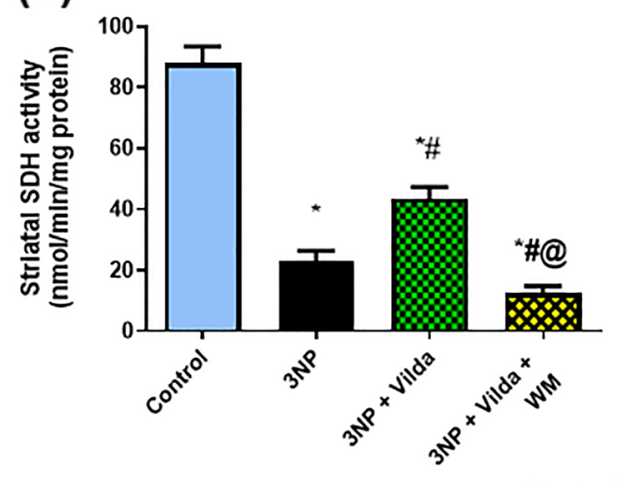

(b)

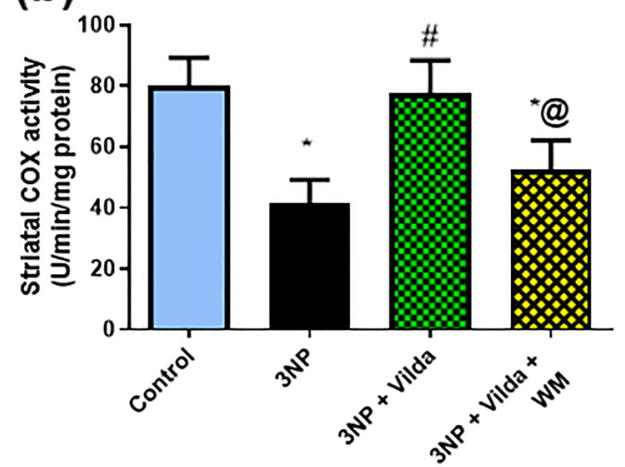

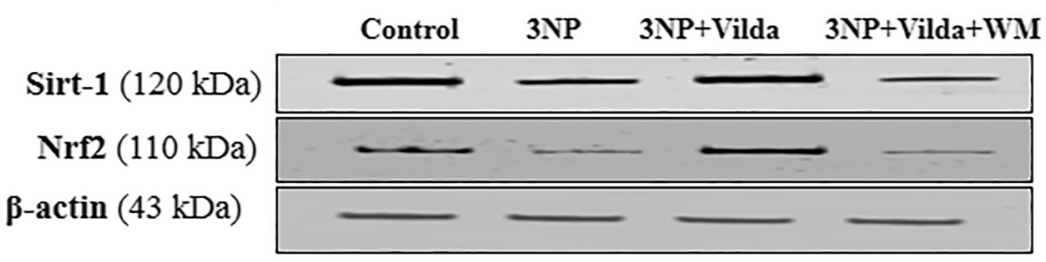

(c)

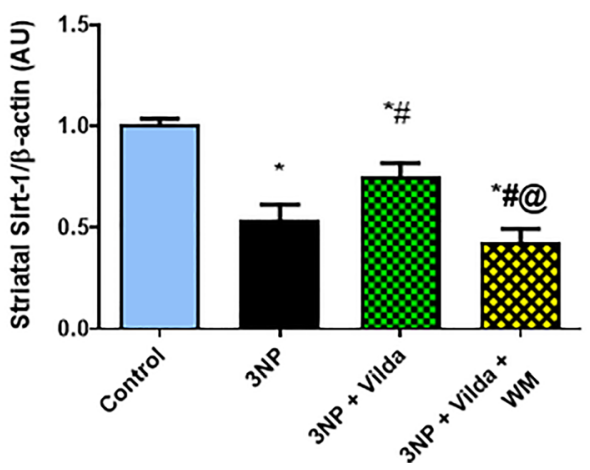

Fig. 3 Effect of Vilda on striatal SDH (a) and COX (b) activities as well as protein expressions of Sirt1 (c) as well Nrf2 (d) in 3NP rats. Each bar with vertical line represents mean \pm S.D. of 5 rats per group. The same membranes were reprobed several times. * vs control, \# vs 3NP, @ (d)

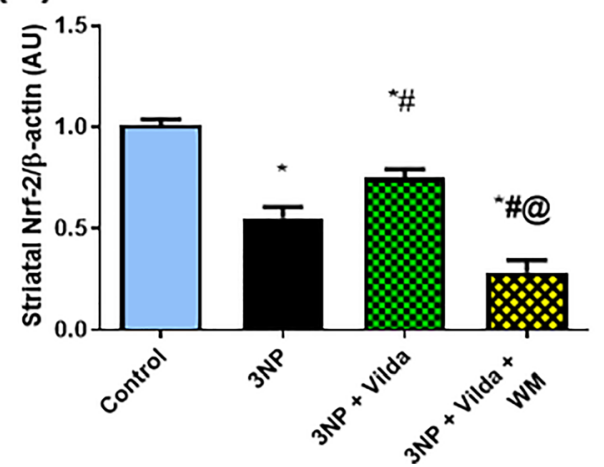

vs Vilda using one-way ANOVA followed by Tukey's post hoc test; $p<0.05$. 3NP; 3-nitropropionic acid, Vilda; vildagliptin, WM; wortmannin 

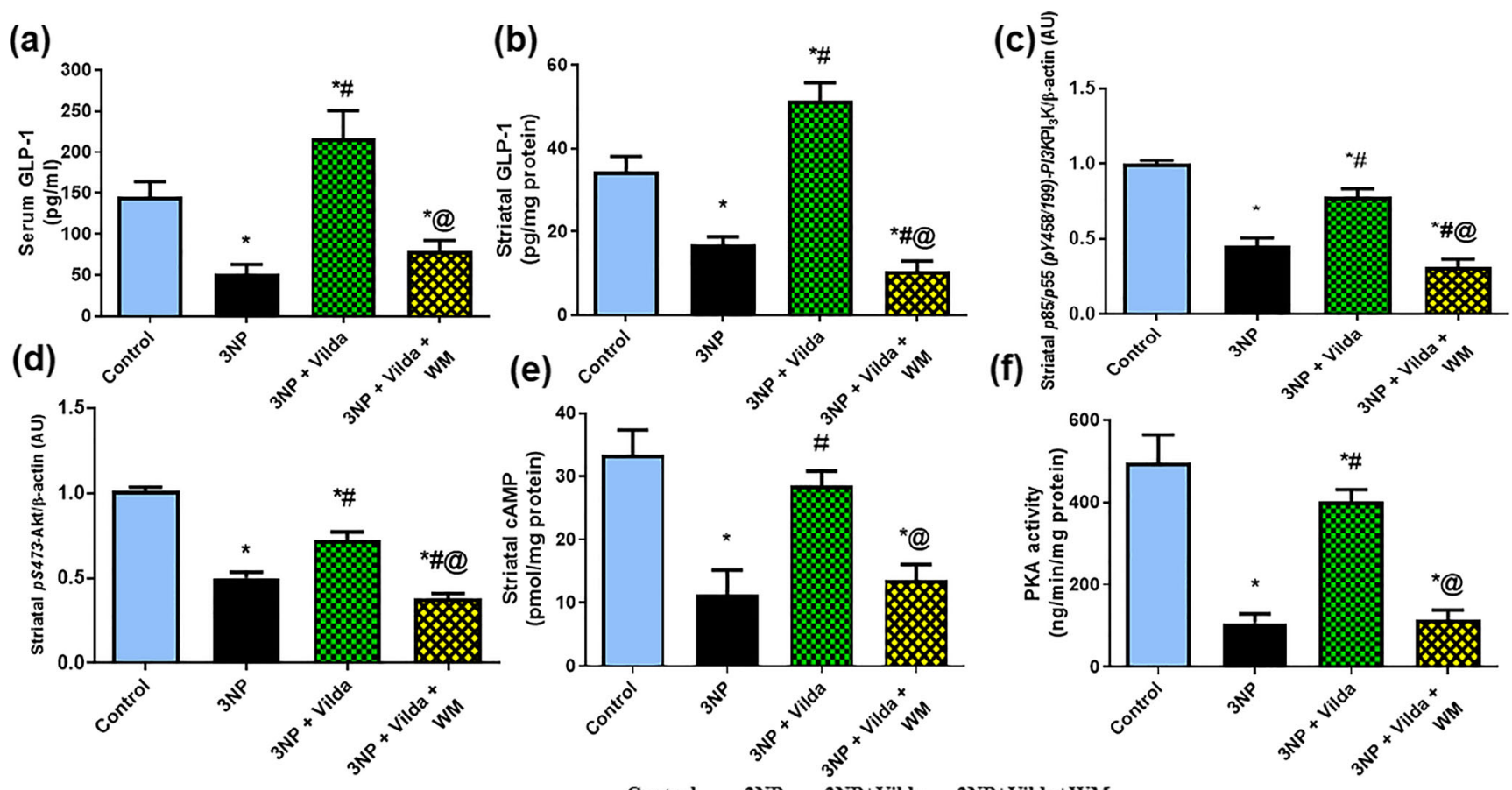

p85/p55 (pY458/199)-PI3K (85 kDa)

pS473-Akt $(60 \mathrm{kDa})$

$\beta$-actin $(43 \mathrm{kDa})$

Fig. 4 Effect of Vilda on serum (a) and striatal (b) GLP-1 level, survival PI3K (c) and Akt (d) protein expressions, cAMP level (e) as well as PKA activity (f) in 3NP rats. Each bar with vertical line represents mean \pm S.D. of 5 rats per group. The same membranes were

almost well-organized striatum region with many apparent intact homogenous distributed neurons with few shrunken degenerated cells, manifested by a $24 \%$ increase in intact neurons, together with apparent intact neuropil with mild infiltration of glial cells. Pre-administration of WM negated Vilda effects and, interestingly, rats from this group showed more widespread core lesion than 3NP rats.

\section{Effect of Vilda on Striatal GFAP Immunoreactivity Alteration in 3NP Rats}

Striatal GFAP immunoreactivity was examined as a principal marker for activation of astrocytes, which are important to the structural and functional support for neurons. As depicted in fig. 10, control rats showed homogenous striatal GFAP distribution with few overall GFAP positive cells. On the other hand, striatal sections from $3 \mathrm{NP}$ rats showed lower numbers of astrocytes inside the lesion core with a dense accumulation of astrocytes outside the lesion core area, with an $11.05 \%$ increase in overall GFAP immunoexpression. Vilda treatment effectively mitigated these effects as indicated by the lower GFAP immunoreactivity (decreased by $6.35 \%$ ) in striata of Vilda-treated rats compared to 3 NP group. Marked astrocytosis similar to that of the $3 \mathrm{NP}$ group was evidenced reprobed several times. * vs control, \# vs 3NP, @ vs Vilda using one-way ANOVA followed by Tukey's post hoc test; p $<0.05$. 3NP; 3 nitropropionic acid, Vilda; vildagliptin, WM; wortmannin

in rats pretreated with WM. Thus, these results suggest that Vilda may provide neuroprotection against 3NP induced toxicity.

\section{Discussion}

In the current study, the presented potential molecular mechanisms revealed the first evidence for the protective role afforded by the DPP-4 enzyme inhibitor, Vilda, in an animal model of 3NP neurotoxicity which mimics the late stages of HD. This notion is supported by multiple lines of evidence: (i) improved cognitive and motor deficits; (ii) enhancement of striatal GLP-1 with the downstream activation of PI3K/Akt pathway; (iii) evoked neurogenesis signals; CREB/BDNF/TrkB besides, PDE10 inhibition; (iv) alleviation of the mitochondrial function and striatal oxidative stress by recovering SDH and COX activities and Sirt1/Nrf2 signaling; (v) elevating the inhibitory GABA and decreasing the excitatory glutamate neurotransmission.

Striatum is the central area in the basal ganglia for motor coordination, as well as, learning and memory functions [26, 27]. 3NP-induced neurodegeneration in striatum causes movement impairment and loss of muscle grip strength, similarly as manifested in the late stages of HD patients [28]. In 
(a)

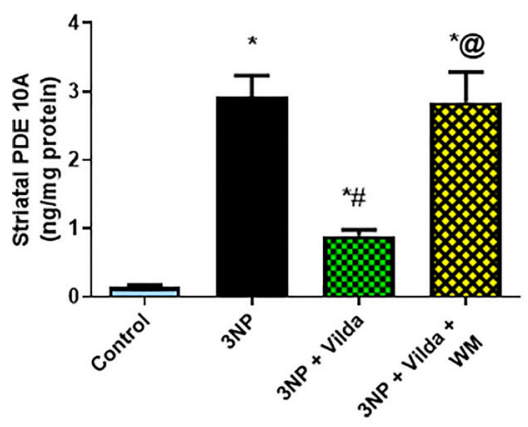

pS133-CREB (43 kDa)

BDNF (14 kDa)

pY515-TrkB (140 kDa)

$\boldsymbol{\beta}$-actin (43 kDa) (b)

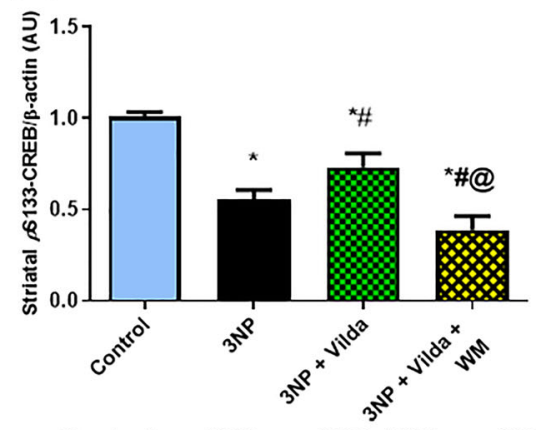

Control 3NP 3NP+Vilda 3NP+Vilda+WM

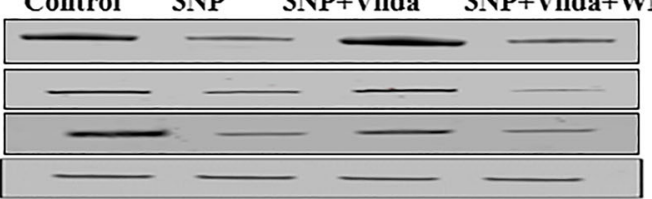

(d)

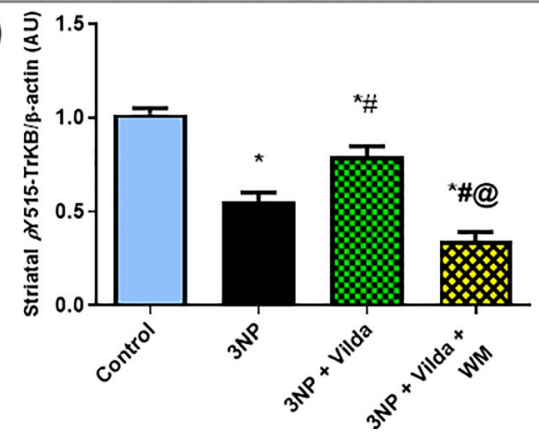

Fig. 5 Effect of Vilda on protein expressions of striatal PDE10A level (a) as well as pCREB (b) BDNF (c) and pTrkB (d) protein expression in 3NP rats. Each bar with vertical line represents mean \pm S.D. of 5 rats per group.

the current study, open field, rotarod performance and grip strength, as well as, Morris water maze tests depicted the behavioral and motor abnormalities of $3 \mathrm{NP}$ intoxication. Nevertheless, Vilda treatment significantly improved muscular strength, locomotor activity, spatial learning and memory skills of 3 NP rats. In this context, Vilda reversed learning and memory defects in a rat model of $\mathrm{AD}$, as well in STZ-induced type 2 diabetic rat model [14, 29]. Likewise, Vilda was reported to improve motor deficits in a rat rotenone model of PD [30].

Fig. 6 Effect of Vilda on striatal GABA (a) and glutamate (b) levels in 3NP rats. Each bar with vertical line represents mean \pm S.D. of 5 rats per group. * vs control, \# vs 3NP, @ vs Vilda using one-way ANOVA followed by Tukey's post hoc test; $\mathrm{p}<0.05$. 3NP; 3-nitropropionic acid, Vilda; vildagliptin, WM; wortmannin (a)

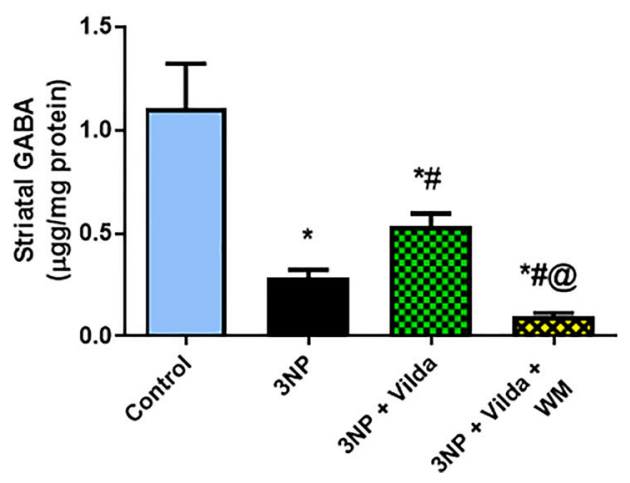

(c)

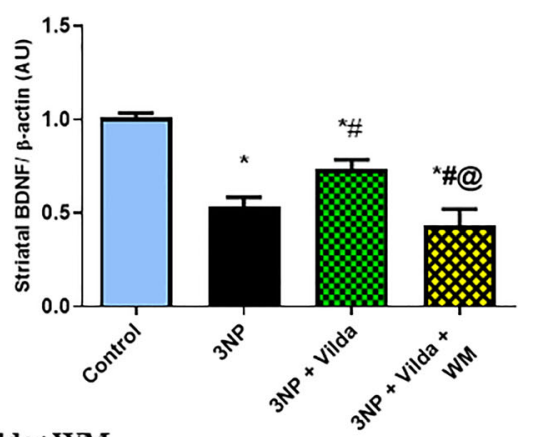

The same membranes were reprobed several times. * vs control, \# vs 3NP, @ vs Vilda using one-way ANOVA followed by Tukey's post hoc test; $p<0.05$. 3NP; 3-nitropropionic acid, Vilda; vildagliptin, WM; wortmannin

In addition, data of the present study revealed that $3 \mathrm{NP}$ induced a significant decrease in the final body weight. This induced body weight loss could be explained on the basis of metabolic dysfunction, including impairment of energy metabolism in animals [31]. Moreover, anorexia and decreased food intake associated with motor deficit could not be excluded as contributing factors to weight loss [24]. Interestingly, Vilda treatment did not cause any further change in body weight, compared to $3 \mathrm{NP}$ group. This effect may be related to the weight neutrality exerted by Vilda which results, in part,

(b)

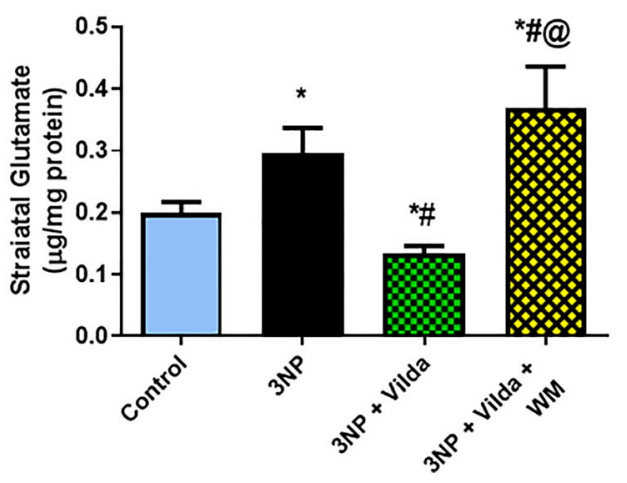


Fig. 7 Effect of Vilda on striatal MDA (a) and GSH (b) levels as well as SOD activity (c) activity in $3 \mathrm{NP}$ rats. Each bar with vertical line represents mean \pm S.D. of 5 rats per group. * vs control, \# vs 3NP, @ vs Vilda using one-way ANOVA followed by Tukey's post hoc test; $\mathrm{p}<0.05$. 3NP; 3-nitropropionic acid, Vilda; vildagliptin, WM; wortmannin

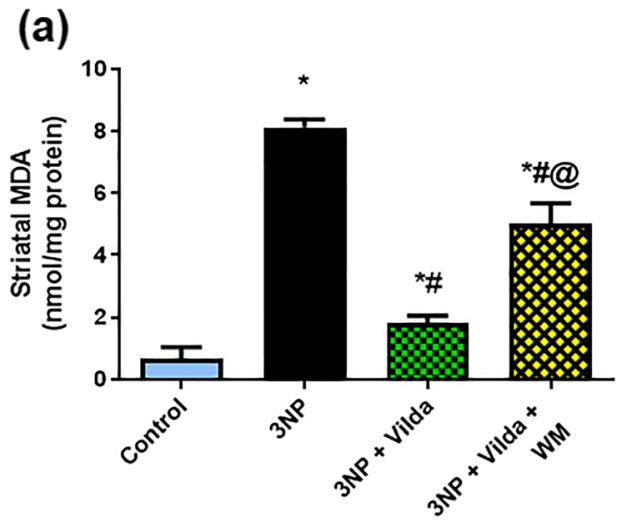

(b)
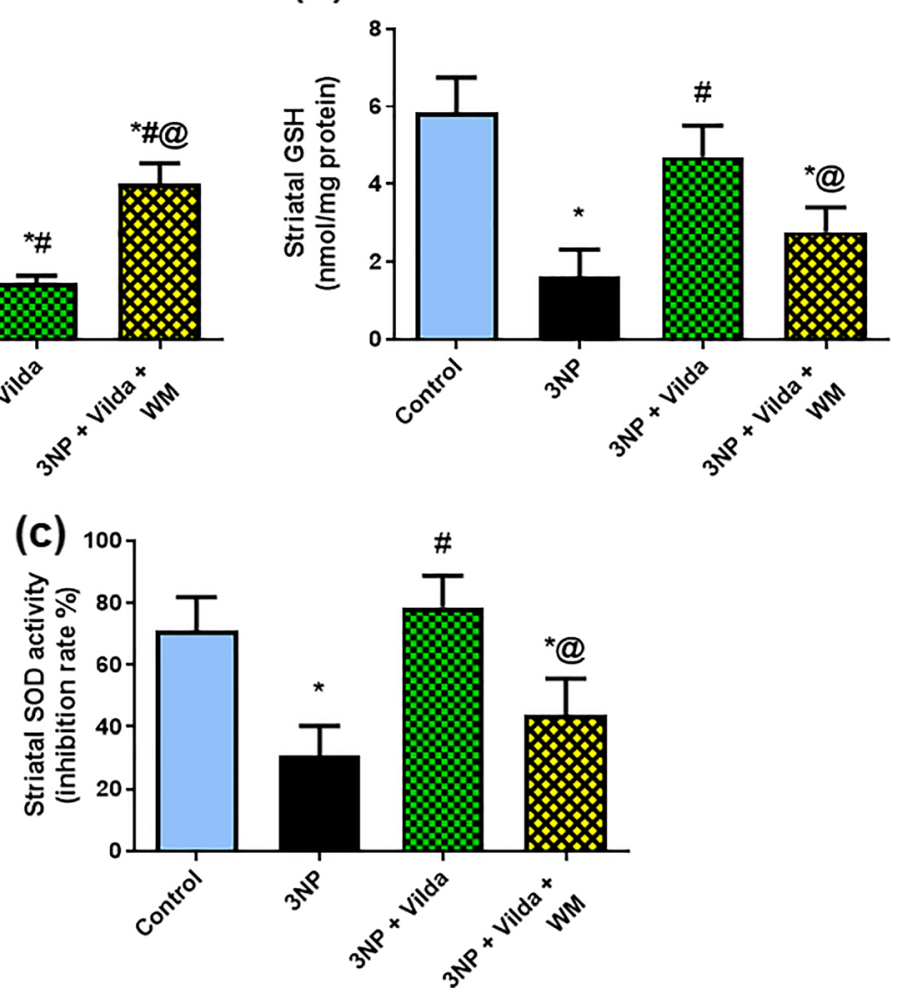

from its intrinsically low risk for hypoglycemia. It is worth mentioning that administration of GLP-1 and GLP-1 analogues (exenatide and liraglutide, for instance) have significant central and metabolic effects on gastric emptying, appetite and food intake, which are translated into significant reductions in body weight [32,33], opposite to neutral effects on body weight with the DPP-4 inhibitors $[34,35]$. The weight neutrality seen with Vilda in several studies $[36,37]$ seems to be a class effect, because the DPP-4 inhibitors, saxagliptin and sitagliptin, have also been shown to produce improvements in glycemic control without significant change in body weight

$[38,39]$. This favorable weight profile, we believe, entitles Vilda superior to other incretin-based therapies, especially where weight loss is a serious complication.

The role of GLP-1 and its receptor in the CNS has been thoroughly studied regarding cellular protection, proliferation and differentiation into neurons. GLP-1 activates adenylyl cyclase, and increases the intracellular cAMP level, which, in turn, activates protein kinase A (PKA), as well as, PI3K, causing phosphorylation and activation of downstream signaling pathways $[5,40]$. Accumulating body of evidence, in cell culture and rodent models, suggests that GLP-1 protects
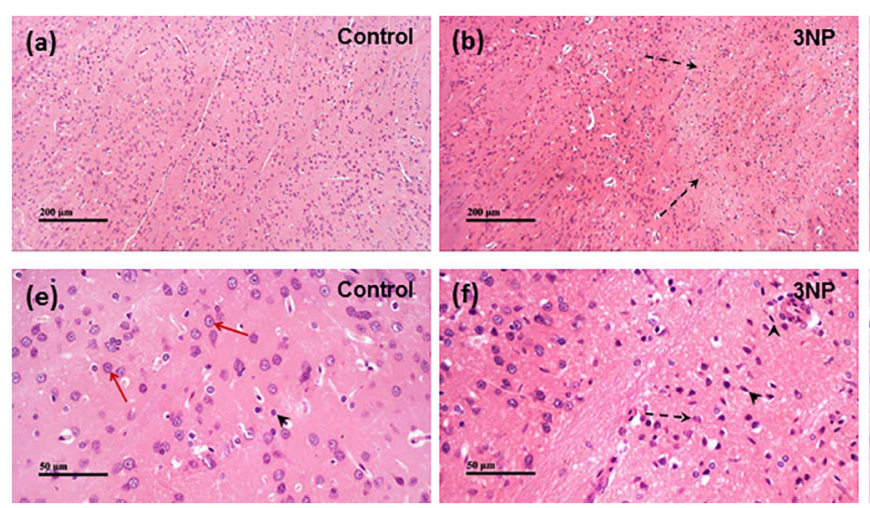

Fig. 8 Effect of Vilda on histopathological alterations in 3NP treated rats. Representative photomicrographs illustrating H\&E staining of the striatum from a,e control group, b,f $3 \mathrm{NP}$ group, $\mathbf{c}, \mathbf{g}$ Vilda-treated group and $\mathbf{d , h}$ WM-treated group. Red arrows indicate intact well organized
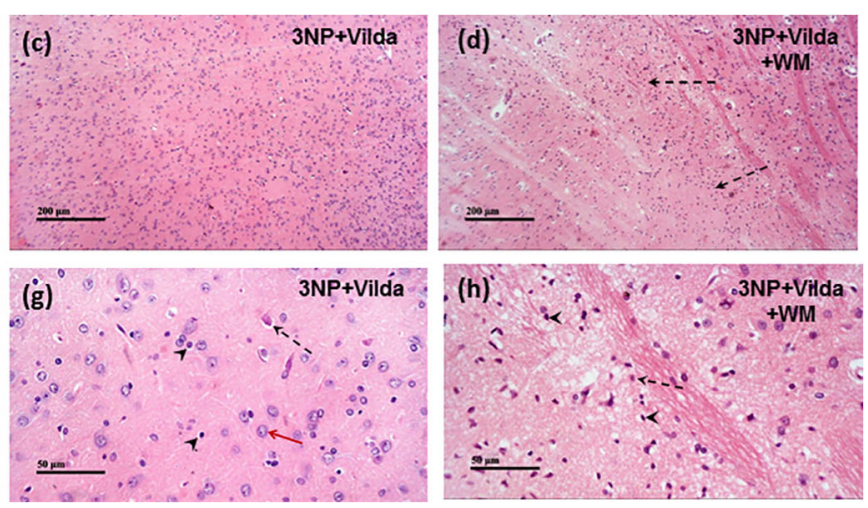

neurons, arrow heads indicate glial cells infiltration and dashed arrows indicate degenerated neurons. Magnifications: $\times 100$ (a-d) $\& \times 400$ (e-h). 3NP; 3-nitropropionic acid, Vilda; vildagliptin, WM; wortmannin 

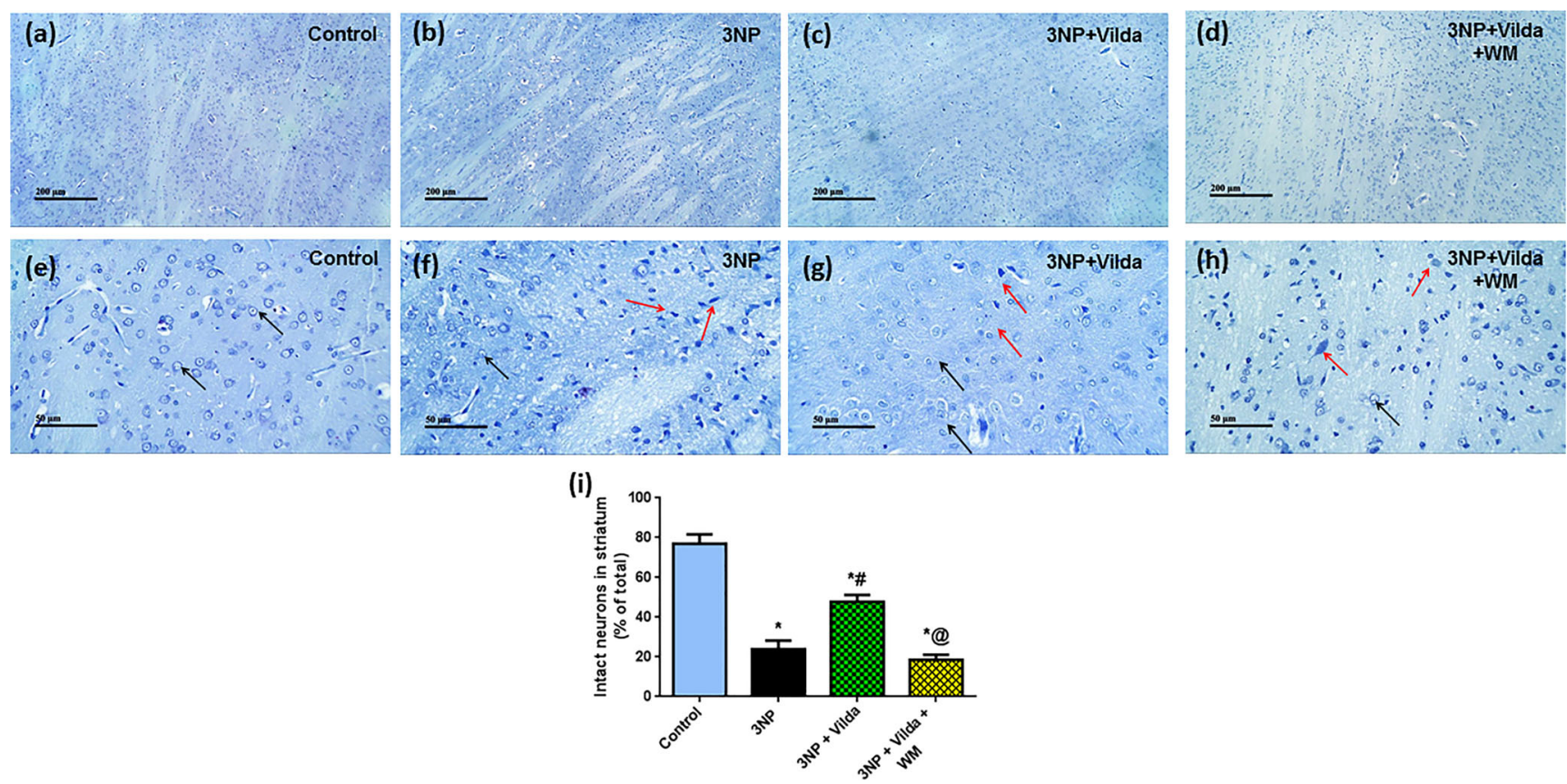

Fig. 9 Effect of Vilda on neuronal survival in 3NP rats. Representative cresyl violet-stained striatal sections from a,e control group, b,f $3 \mathrm{NP}$ group, $\mathbf{c}, \mathbf{g}$ Vilda-treated group and $\mathbf{d}, \mathbf{h}$ WM-treated group. Black arrows indicate intact neurons while red arrows indicate pyknotic changes. Magnifications: $\times 100$ (a-d) $\& \times 400$ (e-h). Panel i depicts the

against neuronal degeneration in experimental models of PD, multiple sclerosis, and AD [41-43]. In the current study, the concurrence of reduced striatal GLP-1 along with striatal damage and neuronal loss, indicated by strong GFAP expression and Nissl staining, provides the first direct evidence for a role of declined striatal GLP-1 level/signaling in 3NP pathology. Such effect was reversed by Vilda treatment which enhanced both serum and striatal GLP-1 levels together with PI3K/Akt phosphorylation. This could be directly linked to inhibition of DPP-4 enzyme, which is a major catabolic enzyme of GLP-1, by Vilda, hence increasing the active GLP-1 levels in the peripheral blood, which then diffuses into the brain [30].

In this context, the neurotrophic and protective actions of GLP-1 against $\mathrm{H}_{2} \mathrm{O}_{2}$-induced oxidative stress and apoptosis, in human neuroblastoma SH-SY5Y cells with overexpressed GLP-1R, were linked to PKA and PI3K signaling enhancement. These results were further confirmed by the use of PI3K inhibitor, LY294002, which markedly attenuated GLP-1 mediated neurotrophic effects [44]. Our results are also in tune with Zhu et al. (2016), who stated the in vivo neuroprotective action of liraglutide, against ischemia-induced apoptosis that was mediated via PI3K/Akt signaling [45]. Noteworthy, WM, a PI3K inhibitor, blocked phosphorylation of PI3K and Akt in Vilda-treated rats, which was concomitant with abolishment of histological amendments afforded by the drug. These results suggest that Vilda neuroprotective effects are mediated, at least in part, through activating PI3K and its downstream target Akt. number of intact neurons in striatal sections of the four groups as $\%$ of total. Each bar with vertical line represents mean \pm S.D. of 3 rats per group. * vs control, \# vs 3NP, @ vs Vilda using one-way ANOVA followed by Tukey's post hoc test; $\mathrm{p}<0.05$. 3NP; 3-nitropropionic acid, Vilda; vildagliptin, WM; wortmannin

To ensure that the previous alterations were directly related to GLP-1 receptor activation, it was necessary to measure cAMP level and PKA activity, as they represent important downstream singling molecules of GLP-1 receptor pathway. Herein, the present results demonstrated that cAMP level and PKA activity were depressed in animals treated with 3NP, which was reversed upon treatment with Vilda. In harmony, it was previously verified that GLP-1 exerts its actions through a $\mathrm{G}$ protein-coupled receptor (GPCR) linked to activation of PKA signaling [46]. On the other hand, the antinecrotic action of GLP-1 in a mouse insulinoma cell line, was abolished by the simultaneous exposure of cells to both PKA and PI3K signaling pathway inhibitors [47].

In addition to the previous data, the canonical pathway, $\mathrm{PI} 3 \mathrm{~K} / \mathrm{Akt}$, is the upstream activator of CREB, that thereafter improves BDNF expression [48]. BDNF binds to TrkB receptor that in turn autophosphorylates tyrosine residues and activates PI3K/Akt pathway [49], thus establishing a mutual activation between PI3K/Akt and CREB/BDNF/TrkB. The $\mathrm{CREB} / \mathrm{BDNF} / \mathrm{TrkB}$ signaling plays a crucial role in synaptic development and plasticity, as evidenced in neurodegenerative diseases that accompanied by abnormal BDNF regulation [50]. This, in turn, rationalizes the reduction of PI3K/Akt phosphorylation in part due to the reduction of GLP-1 and/or $\mathrm{BDNF} /$ TrkB signaling. In the present study, 3NP intoxication showed low protein levels of pCREB, BDNF and TrkB. This occurs in pathogenic HD mouse models and in cells with the mutant huntingtin form where reduced BDNF and TrkB levels 

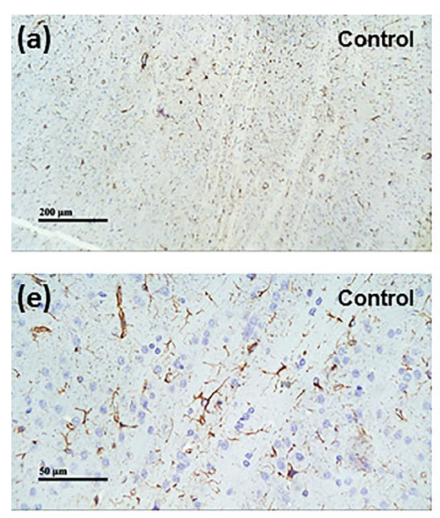
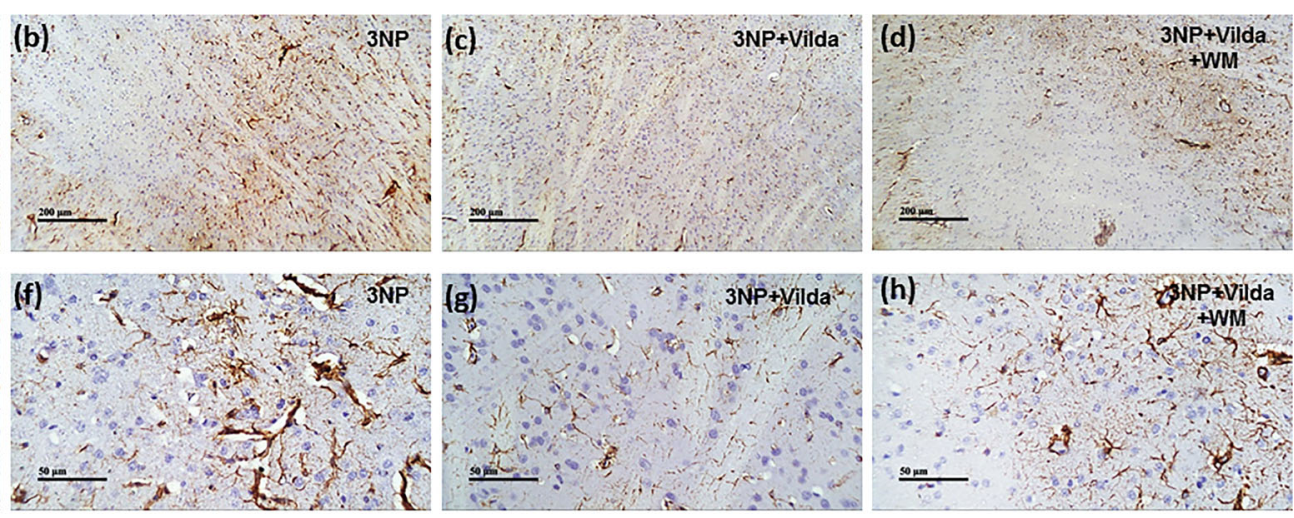

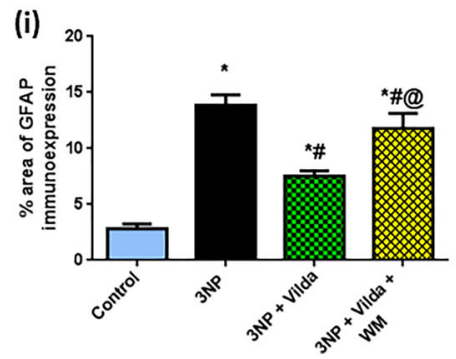

Fig. 10 Effect of Vilda on striatal GFAP immunoreactivity alteration in 3NP rats. Representative photomicrographs of immunohistochemical staining of GFAP in striatum from a,e control group, $\mathbf{b}, \mathbf{f} 3 \mathrm{NP}$ group, $\mathbf{c}, \mathbf{g}$ Vilda-treated group and d,h WM-treated group. Magnifications: $\times 100$ (ad) $\& \times 400$ (e-h). i GFAP optical density of $\%$ area of GFAP

are found. Wild-type huntingtin sequesters the neuronrestrictive silencing factor (NRSF), a huntingtin interaction protein in the cytoplasm that increases the level of BDNF expression. Conversely, mutant huntingtin is associated with a reduction in BDNF, possibly, because of NRSF repression of BDNF transcription [51]. The previous data emphasize the strong link between BDNF levels and HD pathogenesis. Herein, the case was reversed upon treatment with Vilda. Notably, pretreatment of rats with WM, at a dose that blocked Akt phosphorylation and reduced CREB/BDNF/TrkB levels, remarkably attenuated the Vilda neuroprotective effect. In a STZ-induced type 2 diabetic rat model, Zhang et al. (2018) have reported that Vilda treatment against cognitive deficits, is mediated via enhancing Akt/BDNF/nerve growth factor expression levels [14]. Similarly, the effect of Vilda on hippocampal neurons of AD rat model was identified, through targeting the Akt/GSK3 $\beta$ pathway, reducing tau phosphorylation [52].

Interestingly, disturbances in CREB signaling, another downstream target of Akt, have been identified as another culprit in HD pathology. Studies carried on HD patients have verified the decreased transcription of CREB-dependent genes may be related to a deficiency in cAMP. This is supported by the presence of low cAMP in the cerebral spinal fluid of HD patients [53]. Moreover, treatment of mutant huntingtininduced neuronal PC12 cell lines with either cAMP or forskolin, an activator of adenylyl cyclase, induced neurite outgrowth [54]. It is worth mentioning that the magnitude of cAMP is determined by PDE enzyme. Especially in CNS, immunoexpression in striatal sections of the four groups. Each bar with vertical line represents mean \pm S.D. of 3 rats per group. * vs control, \# vs 3NP, @ vs Vilda using one-way ANOVA followed by Tukey's post hoc test; $\mathrm{p}<0.05$. 3NP; 3-nitropropionic acid, Vilda; vildagliptin, WM; wortmannin

PDE10A is abundantly expressed in striatum and plays a role in controlling cAMP/PKA signaling cascades [55]. Needless to say, PDE inhibitors have been demonstrated successfully in HD models to increase cAMP as long PKA and subsequently CREB with BDNF levels [56]. In context, PDE10 inhibition, by papaverine, was found to improve object recognition and spatial memories in R6/1 mice, which may be related, at least in part, to increased CREB phosphorylation levels [55]. Herein, for the first time, the present results demonstrate the inhibitory effect of Vilda on PDE10A. Our results concur with the study conducted with Giampà et al. (2009), who depicted the beneficial effect of treatment with the specific PDE10A inhibitor, TP10, in a quinolinate-induced striatal excitotoxicity rat model. They proposed that the effects of PDE10A inhibition on neuron survival are both indirect, due to increased number of surviving neurons and direct, due to increased neuronal CREB-mediated signaling, which consequently enhanced BDNF induction [57].

Hereafter, the study revealed that the downstream signaling of CREB was not restricted to BDNF regulation but also extended to suppress mitochondrial dysfunction, oxidative stress and neurotransmission imbalance; the cornerstones for the development of HD lesions.

Regarding mitochondrial dysfunction, it is one of the hallmarks of HD, as well a key feature of 3NP HD model. Inevitably, 3NP-treated group showed a significant decline in SDH (complex II) and the terminal enzyme of the respiratory chain; COX (complex IV) activities; reflecting the 
dysregulated mitochondrial function. Contrariwise, Vilda treatment mitigated 3NP's effect and caused a significant increase in SDH and COX activities, thus, restoring the redox balance. Formerly, in high fat diet-induced obese rats, Vilda attenuated brain mitochondrial dysfunction through decreasing brain mitochondrial membrane potential change, brain mitochondrial swelling and exerted antioxidant effects [58]. Likewise, the DPP-4 inhibitor, MK0626, has significantly improved the limited exercise capacity of mice with myocardial infarction through normalization of mitochondrial oxidative capacity and increasing peroxisome proliferator-activated receptor gamma coactivator 1 (PGC-1) and mitochondrial transcription factor A (TFAM) levels, which regulate mitochondrial biogenesis [59]. Considering oxidative stress, one of the direct transcriptional targets of CREB is the enhancement of Sirt1/ Nrf2 signaling pathway that upregulates antioxidant enzymes, scavenges ROS and enhances mitochondrial biogenesis [60]. It is noteworthy that both Sirt1, a NAD ${ }^{+}$dependent protein deacetylase, and $\mathrm{Nrf} 2$, a transcription factor, have remarkable anti-oxidative capacity via activating the expression of promoters containing the antioxidant response element (ARE) resulting in the transcription of ARE-responsive genes which finally quench ROS overproduction [61]. As prementioned for the link between CREB and Sirt1/Nrf2, it was previously demonstrated that CREB deficiency drastically reduces the expression of Sirt1 [62]. On one hand, Sirt1 positively interacts with PGC- $1 \alpha$, thus promoting mitochondrial biogenesis [63], on the other hand, it significantly decreases both Keap 1 expression and Nrf2 ubiquitination, thus promoting the transcriptional activity of Nrf2, and augmenting the protein levels of Nrf2 antioxidant target genes such as SOD, heme oxygenase-1, and glutathione $S$-transferases [64] .

In the current study, the antioxidant activity of Vilda was elaborated by a significant increase in Sirt1 and Nrf2 levels. Also, different DDP-4 inhibitors showed concomitant results where Hee et al. (2015) demonstrated that gemigliptin, in vascular smooth muscle cells of rats, enhanced Nrf2 activity together with increasing Keap1 proteasomal degradation, leading to the induction of Nrf2 target genes [65]. In rat rotenone PD model, Vilda exerted antioxidant potential via reduction of thiobarbituric acid-reactive substances [30].

Additionally, to elaborate the direct effects of Vilda on oxidative stress markers, we estimated the levels of MDA, an endproduct of lipid hydroperoxide, SOD, an enzyme that catalytically reduces $\mathrm{O}_{2}^{-}$to hydrogen peroxide, and finally, GSH, which can catalyze the reduction of hydrogen peroxide. The present results showed that $3 \mathrm{NP}$ promoted oxidative stress as evidenced by the high level of MDA and low levels of SOD activity and reduced GSH, which was reversed by Vilda treatment. This effect may be attributed to Nrf2-dependent upregulation of antioxidative pathways. These findings comply with the study conducted by Atteya H. (2015) that showed that administration of Vilda ameliorated cyclosporine-induced nephrotoxicity in rats through suppressing oxidative stress and modulating levels of reduced GSH, MDA and SOD. Thus, suppression of oxidative stress may be one mechanism by which Vilda exerts its neuroprotective effect [66].

The light spots in neurodegenerative diseases, on the imbalance between GABA and glutamate tone [67]. In the present study, the mitochondrial dysfunction as indicated by lowering SDH and COX activities may provide a validation for the elevated glutamate level in 3NP exposed rats. On the other hand, degeneration of inhibitory GABAergic projection neurons in striatum is a primary event in HD that selectively occurs [68] and evokes neuronal hyperexcitability thereafter. In $3 \mathrm{NP}$ rats of the present study, the same finding occurred and may be accounted to the reduction of BDNF. BDNF/TrkB signaling affects the presynaptic concentration of GABA through activation of GABA transporter (GAT-1) and upregulation of glutamate decarboxylase (GAD) mRNA; the converting enzyme of glutamate to GABA [69]. Parallel data in this regard from Mizuno et al. (1994) related the increase in striatal GABA content, after injection of BDNF into the cerebral ventricles of neonatal rats, to the elevation of GAD activity and GABA uptake activity [70]. Also, Pérez-Navarro et al. (2000) found that intrastriatal grafting of BDNF prevented GAD-positive cell loss in the striata of quinolinate-induced HD disease animal model [71]. Herein, Vildaneuroprotection was evidenced by restoring the neurotransmission balance through a marked reduction in glutamate and elevation in GABA levels, approaching normal levels where this, in part, may be due to Vilda-induced mitochondrial protection and BDNF/TrkB signaling.

Concerning glucose homeostasis, 3NP has been shown to elevate serum glucose level. This effect was formerly attributed to SDH inhibition, which results in less ATP generation, reduced glucose-induced mitochondrial membrane potential hyperpolarization and, subsequently, affecting glucoseinduced alterations in cytosolic $\mathrm{Ca}^{2+}$ concentration and reducing insulin secretion [72]. Interestingly, previous data showed that SDH inhibition by $3 \mathrm{NP}$ impaired insulin secretion via lowering gene transcription of preproinsulin and synthesis of proinsulin [73]. Indeed, Vilda normalized serum glucose level through augmenting glucose stimulated insulin secretion. On the contrary, WM significantly raised serum glucose level which assures that GLP-1-induced glucose uptake is PI3K dependent. WM significantly attenuated GLP-1-mediated increase in GLUT4 protein level [74].

Taken together, the findings of the current study provide the first evidence for the anti-Huntington effect of Vilda for preventing striatal degeneration instigated by $3 \mathrm{NP}$, via conquering GLP-1/PI3K/Akt/CREB/BDNF/TrkB signaling and Sirt1/Nrf2 axis, thus, offering a new perspective for the potential role of DPP-4 inhibition in HD. 
Acknowledgements The authors are grateful to Dr. Mohamed Abdel Razek (Pathology Department, Faculty of Veterinary Medicine, Cairo University, Egypt) for his support and assistance in histopathological examination. Moreover, we would like to thank Tarek Abdel Hamid for aiding us in animal breeding.

\section{Compliance with Ethical Standards}

The protocols used in this study were approved by the Ethics Committee for Animal Experimentation at Faculty of Pharmacy, Cairo University (Permit Number: PT BC2403).

Conflict of Interest The authors declare that they have no conflicts of interest.

\section{References}

1. Ross CA, Aylward EH, Wild EJ, Langbehn DR, Long JD, Warner $\mathrm{JH}$, et al. Huntington disease: Natural history, biomarkers and prospects for therapeutics. Nat Rev Neurol. 2014;10(4):204-16.

2. Novak MJU, Tabrizi SJ. Huntington's disease: Clinical presentation and treatment. In: International Review of Neurobiology. 2011. p. 297-323.

3. Shannon RP. DPP-4 Inhibition and Neuroprotection: Do Mechanisms Matter? Diabetes. 2013;62(4):1029-31.

4. Martin B, Golden E, Carlson OD, Pistell P, Zhou J, Kim W, et al. Exendin-4 Improves Glycemic Control, Ameliorates Brain and Pancreatic Pathologies, and Extends Survival in a Mouse Model of Huntington's Disease. Diabetes.;58(2):318-28.

5. Athauda D, Foltynie T. The glucagon-like peptide 1 (GLP) receptor as a therapeutic target in Parkinson's disease: mechanisms of action. Drug Discov Today. 2016;21(5):802-18.

6. Al-Badri G, Leggio G, Musumeci G, Marzagalli R, Drago F, Castorina A. Tackling dipeptidyl peptidase IV in neurological disorders. Neural Regen Res. 2018;13(1):26.

7. Chalichem NSS, Sai Kiran PSS, Basavan D. Possible role of DPP4 inhibitors to promote hippocampal neurogenesis in Alzheimer's disease. J Drug Target. 2018;26(8):670-5.

8. Nassar NN, Al-Shorbagy MY, Arab HH, Abdallah DM. Saxagliptin: A novel antiparkinsonian approach. Neuropharmacology. 2015;89:308-17.

9. Mathieu C, Degrande E. Vildagliptin: a new oral treatment for type 2 diabetes mellitus. Vasc Health Risk Manag. 2008;4(6):1349-60.

10. Ma Q, Jiang L, Mao J, Xu W, Huang M. Vildagliptin prevents cognitive deficits and neuronal apoptosis in a rat model of Alzheimer's disease. Mol Med Rep. 2017;17(3):4113-9.

11. El-Marasy SA, Abdel-Rahman RF, Abd-Elsalam RM. Neuroprotective effect of vildagliptin against cerebral ischemia in rats. Naunyn Schmiedebergs Arch Pharmacol. 2018;391(10): 1133-45.

12. Chong ZZ, Shang YC, Wang S, Maiese K. A Critical Kinase Cascade in Neurological Disorders: PI 3-K, Akt, and mTOR. Future Neurol. 2012;7(6):733-48.

13. Kumar P, Kalonia H, Kumar A. Expression of Concern: Role of LOX/COX pathways in 3-nitropropionic acid-induced Huntington's Disease-like symptoms in rats: protective effect of licofelone. Br J Pharmacol. 2011;164(2b):644-54.

14. Zhang D, Shi N, Fang H, Ma L, Wu W, Zhang Y, et al. Vildagliptin, a DPP4 inhibitor, alleviates diabetes-associated cognitive deficits by decreasing the levels of apoptosis-related proteins in the rat hippocampus. Exp Ther Med. 2018 15(6):5100-5106.

15. Yue T-L, Bao W, Gu J-L, Cui J, Tao L, Ma X-L, et al. Rosiglitazone treatment in Zucker diabetic Fatty rats is associated with ameliorated cardiac insulin resistance and protection from ischemia/reperfusion-induced myocardial injury. Diabetes. 2005;54(2):554-62.

16. Rabie MA, Abd El Fattah MA, Nassar NN, El-Abhar HS, Abdallah DM. Angiotensin 1-7 ameliorates 6hydroxydopamine lesions in hemiparkinsonian rats through activation of MAS receptor/PI3K/Akt/BDNF pathway and inhibition of angiotensin II type-1 receptor/NF-KB axis. Biochem Pharmacol 2018;151:126-34.

17. Avila DS, Colle D, Gubert P, Palma AS, Puntel G, Manarin F, et al. A possible neuroprotective action of a vinylic telluride against Mninduced neurotoxicity. Toxicol Sci. 2010;115(1):194-201.

18. Jones BJ, Roberts DJ. The quantiative measurement of motor incoordination in naive mice using an acelerating rotarod. J Pharm Pharmacol. 1968;20(4):302-4.

19. Coluccia A, Borracci P, Renna G, Giustino A, Latronico T, Riccio P, et al. Developmental omega-3 supplementation improves motor skills in juvenile-adult rats. Int J Dev Neurosci. 2009;27(6):599605 .

20. Bradford MM. A rapid and sensitive method for the quantitation of microgram quantities of protein utilizing the principle of proteindye binding. Anal Biochem. 1976 May;72(1-2):248-54.

21. Lowry OH, Rosebrough NJ, Farr AL, Randall RJ. Protein measurement with the Folin phenol reagent. J Biol Chem. 1951;193(1): 265-75.

22. ELLMAN GL. Tissue sulfhydryl groups. Arch Biochem Biophys. 1959 May ;82(1):70-7.

23. Sharman EH, Bondy SC. Effects of age and dietary antioxidants on cerebral electron transport chain activity. Neurobiol Aging. 2001;22(4):629-34.

24. Ahmed LA, Darwish HA, Abdelsalam RM, Amin HAA. Role of Rho Kinase Inhibition in the Protective Effect of Fasudil and Simvastatin Against 3-Nitropropionic Acid-Induced Striatal Neurodegeneration and Mitochondrial Dysfunction in Rats. Mol Neurobiol. 2016;53(6):3927-38.

25. Dunnett SB, Carter RJ, Watts C, Torres EM, Mahal A, Mangiarini L, et al. Striatal Transplantation in a Transgenic Mouse Model of Huntington's Disease. Exp Neurol. 1998 Nov 1;154(1):31-40.

26. Graybiel AM, Grafton ST. The Striatum: Where Skills and Habits Meet. Cold Spring Harb Perspect Biol. 2015; 7(8):a021691.

27. Liljeholm M, O'Doherty JP. Contributions of the striatum to learning, motivation, and performance: an associative account. Trends Cogn Sci. 2012;16(9):467-75.

28. Suganya SN, Sumathi T. Effect of rutin against a mitochondrial toxin, 3-nitropropionicacid induced biochemical, behavioral and histological alterations-a pilot study on Huntington's disease model in rats. Metab Brain Dis. 2017;32(2):471-81.

29. Kosaraju J, Murthy V, Basha R, Dubala A. Vildagliptin : an antidiabetes agent ameliorates cognitive deficits and pathology observed in streptozotocin-induced Alzheimer' s disease. 2013;65:1773-84.

30. Abdelsalam RM, Safar MM. Neuroprotective effects of vildagliptin in rat rotenone Parkinson's disease model: role of RAGE-NFKB and Nrf2-antioxidant signaling pathways. J Neurochem. 2015;133(5):700-7.

31. Dhadde SB, Nagakannan P, Roopesh M, Kumar SRA, Thippeswamy BS, Veerapur VP, et al. ScienceDirect Effect of embelin against 3-nitropropionic acid-induced Huntington 's disease in rats. Biomed Pharmacother. 2016;77:52-8.

32. Duarte AI, Sjögren M, Santos MS, Oliveira CR, Moreira PI, Björkqvist M. Dual Therapy with Liraglutide and Ghrelin Promotes Brain and Peripheral Energy Metabolism in the R6/2 Mouse Model of Huntington's Disease. Sci Rep. 2018;8(1):1-14.

33. Foley JE, Jordan J. Weight neutrality with the DPP-4 inhibitor, vildagliptin : Mechanistic basis and clinical experience. Vasc Health Risk Manag. 2010;541-8. 
34. Mudaliar S, Henry RR. Incretin Therapies : Effects Beyond Glycemic Control. Am J Med. 2009;122(6):S25-36.

35. Raun K, Von Voss P, Gotfredsen CF, Golozoubova V, Rolin B, Knudsen LB. Liraglutide, a long-acting glucagon-like peptide-1 analog, reduces body weight and food intake in obese candy-fed rats, whereas a dipeptidyl peptidase-IV inhibitor, vildagliptin, does not. Diabetes. 2007;56(1):8-15.

36. Ferrannini E, Fonseca V, Zinman B, Matthews D, Ahrén B, Byiers $\mathrm{S}$, et al. Fifty-two-week efficacy and safety of vildagliptin vs. glimepiride in patients with type 2 diabetes mellitus inadequately controlled on metformin monotherapy. Diabetes, Obes Metab. 2009;11(2):157-66.

37. Pi-Sunyer FX, Schweizer A, Mills D, Dejager S. Efficacy and tolerability of vildagliptin monotherapy in drug-naïve patients with type 2 diabetes. Diabetes Res Clin Pract. 2007;76(1):132-8.

38. Rosenstock TR, Duarte AI, Rego AC. Mitochondrial-associated metabolic changes and neurodegeneration in Huntington's disease - from clinical features to the bench. Curr Drug Targets. 2010;11(10):1218-36.

39. Nauck MA, Meininger G, Sheng D, Terranella L, Stein PP, Sitagliptin Study 024 Group. Efficacy and safety of the dipeptidyl peptidase-4 inhibitor, sitagliptin, compared with the sulfonylurea, glipizide, in patients with type 2 diabetes inadequately controlled on metformin alone: a randomized, double-blind, non-inferiority trial. Diabetes, Obes Metab. 2007 Mar;9(2):194-205.

40. Marosi K, Mattson MP. BDNF mediates adaptive brain and body responses to energetic challenges. Trends Endocrinol Metab. 2014 Feb;25(2):89-98.

41. Rangasamy SB, Soderstrom K, Bakay RAE, Kordower JH. Neurotrophic factor therapy for Parkinson's disease. In: Progress in brain research. 2010. p. 237-64.

42. McClean PL, Gault VA, Harriott P, Hölscher C. Glucagon-like peptide-1 analogues enhance synaptic plasticity in the brain: A link between diabetes and Alzheimer's disease. Eur J Pharmacol. 2010 Mar 25;630(1-3):158-62.

43. DellaValle B, Brix GS, Brock B, Gejl M, Landau AM, Møller A, et al. Glucagon-Like Peptide-1 Analog, Liraglutide, Delays Onset of Experimental Autoimmune Encephalitis in Lewis Rats. Front Pharmacol. 2016;7:433.

44. Li Y, Tweedie D, Mattson MP, Holloway HW, Greig NH. Enhancing the GLP-1 receptor signaling pathway leads to proliferation and neuroprotection in human neuroblastoma cells. J Neurochem. 2010; 113(6):1621-31.

45. Zhu H, Zhang Y, Shi Z, Lu D, Li T, Ding Y, et al. The Neuroprotection of Liraglutide Against Ischaemia-induced Apoptosis through the Activation of the PI3K/AKT and MAPK Pathways. Sci Rep. 2016;6(1):26859.

46. Drucker DJ, B DPPL, Medicine PLB, Hospital G. Minireview : Glucagon-Like Peptides Regulate Cell. Endocrinology. 2015;145: 2653-9.

47. Perfetti R, Endocrinology D, Metabolism HH, Z DCX, Medical C. Glucagon-Like Peptide-1 Inhibits Apoptosis of Phosphatidylinositol 3-Kinase-Dependent Pathway. Endocrinology. 2003;144(4):1444-55.

48. Zuo D, Lin L, Liu Y, Wang C, Xu J, Sun F, et al. Baicalin Attenuates Ketamine-Induced Neurotoxicity in the Developing Rats: Involvement of PI3K/Akt and CREB/BDNF/Bcl-2 Pathways. Neurotox Res. 2016;30(2):159-72.

49. Hu Y, Russek SJ. BDNF and the diseased nervous system: a delicate balance between adaptive and pathological processes of gene regulation. J Neurochem. 2008;105(1):1-17.

50. Yoshii A, Constantine-Paton M. Postsynaptic BDNF-TrkB signaling in synapse maturation, plasticity, and disease. Dev Neurobiol. 2010; 70(5):304-22.

51. Zuccato C, Tartari M, Crotti A, Goffredo D, Valenza M, Conti L, et al. Huntingtin interacts with REST/NRSF to modulate the transcription of NRSE-controlled neuronal genes. Nat Genet. 2003;35(1):76-83.

52. Ma Q, Jiang L, Mao J, Xu W, Huang M. Vildagliptin prevents cognitive deficits and neuronal apoptosis in a rat model of Alzheimer's disease. Mol Med Rep. 2018;17(3):4113-9.

53. Gines S, Seong IS, Fossale E, Ivanova E, Trettel F, Gusella JF, et al. Specific progressive cAMP reduction implicates energy deficit in presymptomatic Huntington's disease knock-in mice. Hum Mol Genet. 2003;12(5):497-508.

54. Wyttenbach A. Polyglutamine expansions cause decreased CREmediated transcription and early gene expression changes prior to cell death in an inducible cell model of Huntington's disease. Hum Mol Genet. 2001;10(17):1829-45.

55. Giralt A, Saavedra A, Carretón O, Arumí H, Tyebji S, Alberch J, et al. PDE10 inhibition increases GluA1 and CREB phosphorylation and improves spatial and recognition memories in a Huntington's disease mouse model. Hippocampus. 2013;23(8): 684-95.

56. Fusco FR, Giampà C. Phosphodiesterases as therapeutic targets for Huntington's disease. Curr Pharm Des. 2015;21(3):365-77.

57. Giampà $\mathrm{C}$, Patassini $\mathrm{S}$, Borreca $\mathrm{A}$, Laurenti $\mathrm{D}$, Marullo $\mathrm{F}$, Bernardi $\mathrm{G}$, et al. Phosphodiesterase 10 inhibition reduces striatal excitotoxicity in the quinolinic acid model of Huntington's disease. Neurobiol Dis . 2009;34(3):450-6.

58. Sa-nguanmoo P, Tanajak P, Kerdphoo S, Jaiwongkam T. SGLT2inhibitor and DPP-4 inhibitor improve brain function via attenuating mitochondrial dysfunction, insulin resistance, in fl ammation, and apoptosis in HFD-induced obese rats. Toxicol Appl Pharmacol. 2017;333:43-50.

59. Takada S, Masaki Y, Kinugawa S, Matsumoto J, Furihata T, Mizushima W, et al. Dipeptidyl peptidase-4 inhibitor improved exercise capacity and mitochondrial biogenesis in mice with heart failure via activation of glucagon-like peptide-1 receptor signalling. 2016;111:338-47.

60. Ding YW, Zhao GJ, Li XL, Hong GL, Li MF, Qiu QM, et al. SIRT1 exerts protective effects against paraquat-induced injury in mouse type II alveolar epithelial cells by deacetylating NRF2 in vitro. Int J Mol Med. 2016;37(4):1049-58.

61. Satta S, Mahmoud AM, Wilkinson FL, Yvonne Alexander M, White SJ. The Role of Nrf2 in Cardiovascular Function and Disease. Oxid Med Cell Longev. 2017;1-18.

62. Lagouge M, Argmann C, Gerhart-Hines Z, Meziane H, Lerin C, Daussin $\mathrm{F}$, et al. Resveratrol improves mitochondrial function and protects against metabolic disease by activating SIRT1 and PGC1alpha. Cell. 2006;127(6):1109-22.

63. Huang K, Gao X, Wei W. The crosstalk between Sirt1 and Keap1/ Nrf2/ARE anti-oxidative pathway forms a positive feedback loop to inhibit FN and TGF- $\beta 1$ expressions in rat glomerular mesangial cells. Exp Cell Res. 2017;361(1):63-72.

64. Benarroch EE. Nrf2, cellular redox regulation, and neurologic implications. Neurology [Internet]. 2017;88(20):1942-50.

65. Hee S, Park S, Joo C, Leem J, Park K, Lee I. Dipeptidyl peptidase-4 inhibition by gemigliptin prevents abnormal vascular remodeling via NF-E2-related factor 2 activation. Vascul Pharmacol. 2015;1-9.

66. Ateyya H. International Immunopharmacology Amelioration of cyclosporine induced nephrotoxicity by dipeptidyl peptidase inhibitor vildagliptin. Int Immunopharmacol. 2015;28:571-7.

67. Guerriero RM, Giza CC, Rotenberg A. Glutamate and GABA imbalance following traumatic brain injury. Curr Neurol Neurosci Rep. 2015;15(5):27.

68. Pineda R, Canals JM, Bosch M, Adell A, Mengod G, Artigas F, et al. Brain-derived neurotrophic factor modulates dopaminergic deficits in a transgenic mouse model of Huntington' s disease. J Neurochem. 2005;93:1057-68.

69. Vaz SH, Jørgensen TN, Cristóvão-Ferreira S, Duflot S, Ribeiro JA, Gether U, et al. Brain-derived Neurotrophic Factor (BDNF) 
Enhances GABA Transport by Modulating the Trafficking of GABA Transporter-1 (GAT-1) from the Plasma Membrane of Rat Cortical Astrocytes. J Biol Chem. 2011;286(47):40464-76.

70. Mizuno K, Carnahan J, Nawa H. Brain-Derived Neurotrophic Factor Promotes Differentiation of Striatal GABAergic Neurons. Dev Biol. 1994;165(1):243-56.

71. Pérez-Navarro E, Canudas AM, Akerund P, Alberch J, Arenas E. Brain-derived neurotrophic factor, neurotrophin-3, and neurotrophin- $4 / 5$ prevent the death of striatal projection neurons in a rodent model of Huntington's disease. J Neurochem. 2000;75(5):2190-9.

72. Edalat A, Schulte-Mecklenbeck P, Bauer C, Undank S, KrippeitDrews P, Drews G, et al. Mitochondrial succinate dehydrogenase is involved in stimulus-secretion coupling and endogenous ROS formation in murine beta cells. Diabetologia. 2015;58(7):1532-41.

73. Leibowitz G, Khaldi MZ, Shauer A, Parnes M, Oprescu AI, Cerasi E, et al. Mitochondrial regulation of insulin production in rat pancreatic islets. Diabetologia. 2005;48(8):1549-59.

74. Green CJ, Henriksen TI, Pedersen BK, Solomon TPJ. Glucagon Like Peptide-1-Induced Glucose Metabolism in Differentiated Human Muscle Satellite Cells Is Attenuated by Hyperglycemia. PLoS One. 2012;7(8).

Publisher's Note Springer Nature remains neutral with regard to jurisdictional claims in published maps and institutional affiliations. 